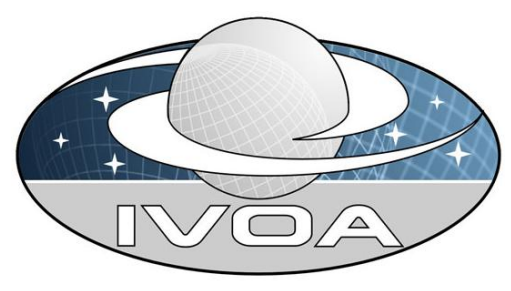

International

Virtual

Observatory

Alliance

\title{
Data Model for Astronomical DataSet Characterisation Version 1.13
}

\section{IVOA Recommendation 2008 March 25}

\author{
This version: \\ http://www. ivoa.net/Documents/REC/DM/CharacterisationDM-20080325. \\ pdf
}

\section{Latest version:}

http://www.ivoa.net/Documents/latest/CharacterisationDM.html

\section{Previous versions:}

1.12

\section{Editor(s):}

Mireille Louys, Anita Richards, François Bonnarel, Alberto Micol, Igor Chilingarian, Jonathan McDowell

\section{Authors:}

IVOA Data Model Working Group

\section{Abstract}

This document defines the high level metadata necessary to describe the physical parameter space of observed or simulated astronomical data sets, such as 2D-images, data cubes, X-ray event lists, IFU data, etc.. The Characterisation data model is an abstraction which can be used to derive a structured description of any relevant data and thus to facilitate its discovery and scientific interpretation. The model aims at facilitating the manipulation of heterogeneous data in any VO framework or portal. 
A VO Characterisation instance can include descriptions of the data axes, the range of coordinates covered by the data, and details of the data sampling and resolution on each axis. These descriptions should be in terms of physical variables, independent of instrumental signatures as far as possible.

Implementations of this model has been described in the IVOA Note available at:

http://www.ivoa.net/Documents/latest/ImplementationCharacterisation.html

Utypes derived from this version of the UML model are listed and commented in the following IVOA Note:

http://www . ivoa.net/Documents/latest/UtypeListCharacterisationDM.html

An XML schema has been build up from the UML model and is available at:

http://www.ivoa.net/xml/Characterisation/Characterisation-v1.11.xsd

\section{Status of this document}

This document has been produced by the Data Model Working Group. It has been reviewed by IVOA Members and other interested parties, and has been endorsed by the IVOA Executive Committee as an IVOA Recommendation. It is a stable document and may be used as reference material or cited as a normative reference from another document. IVOA's role in making the Recommendation is to draw attention to the specification and to promote its widespread deployment. This enhances the functionality and interoperability inside the Astronomical Community.

\section{Acknowledgements}

Members of the IVOA Data Model Working Group, including representatives of the US NVO, Astrogrid, and the Euro-VO have contributed to the present draft. The editors particularly acknowledge the input from P. Didelon, G. Lemson, A. Rots, L. Shaw, B. Thomas and D. Tody. F. Bonnarel and M. Louys acknowledge support from the French ACI-GRID project, IDHA and the EU-funded VOTech project. 


\section{Contents}

1 Status of this document 2

2 Introduction $\quad \mathbf{5}$

2.1 The purpose of the Characterisation model . . . . . . . . . 5

2.2 Scope of the document . . . . . . . . . . . . . . . 6

2.3 Links to other IVOA modeling efforts . . . . . . . . . . 7

3 Exploring the Characterisation concepts $\quad 7$

3.1 Overview: a geometric approach . . . . . . . . . . 7

3.2 Examples of Characterisation . . . . . . . . . . . . . . 9

3.3 Structure and development strategy . . . . . . . . . . . . . 9

3.4 The Axis point of view . . . . . . . . . . . . . . . . . . 9

3.4 .1 Axes and their attributes . . . . . . . . . . 9

3.4 .2 Axes flags . . . . . . . . . . . . . . . 11

3.5 Accuracy . . . . . . . . . . . . . . . . . 11

3.6 The Property point of view . . . . . . . . . . . . . 11

3.6.1 Coverage . . . . . . . . . . . . . . . 12

3.6.2 Resolution and Sampling Precision . . . . . . . . . . 14

3.7 Presentation of layered information . . . . . . . . . . . . . 15

4 The Model $\quad 15$

4.1 The role and structure of the Model . . . . . . . . . . . . 15

4.2 Axis description . . . . . . . . . . . . . . . . 16

4.2.1 Flags and other qualifying information . . . . . . . . 18

4.2.1.1 Independent or dependent status . . . . . . . 18

4.2.1.2 Calibration status . . . . . . . . . 18

4.2.1.3 Sampling status . . . . . . . . . . . . 19

4.2.2 Errors in Characterisation: the Accuracy class . . . . . 19

4.3 Navigation in the model: by axis or by properties? . . . . . . . 19

4.4 Implementing the model using STC elements . . . . . . . . . . 21

5 XML Serialization $\quad 23$

5.1 XML schema (Axis First) . . . . . . . . . . . . . . . 23

5.1.1 Design of the schema . . . . . . . . . . . 23

5.1.2 Building blocks of the schemata . . . . . . . . . 24

5.2 Utypes generation: select one ordering strategy . . . . . . . . 31

5.2 .1 VOTABLE serialisation . . . . . . . . . 31

A Appendix A: XML serialisation example 31 
C Appendix C: Characterisation of various dataset properties 32

D Appendix D: Requirements for Data Model compliance 38

D.1 Limitations in this version . . . . . . . . . . . . . . . 38

D.2 Implementing Characterisation . . . . . . . . . . . . 38

D.2.1 Data Providers . . . . . . . . . . . . 38

D.3 Requirements for compliance . . . . . . . . . . . . . . 39

D.3.1 General considerations . . . . . . . . . . . . 39

D.3.2 Defaults . . . . . . . . . . . . . 39

D.4 Axes . . . . . . . . . . . . . . . . . 40

D.4.1 Axis Flags . . . . . . . . . . . . . . . 40

D.5 Coverage . . . . . . . . . . . . . . . . . . . 41

D.6 Other Properties: Resolution and Sampling Precision . . . . . 41

D.6.1 Resolution . . . . . . . . . . . . . . . . . 42

D.6.2 Sampling Precision . . . . . . . . . . . . . . . 42

D.7 Accuracy . . . . . . . . . . . . . . . . . . . . . . . . 42

E Appendix E: Updates of this document 43 


\section{Introduction}

This document defines an abstract data model called "Data Set Characterisation" (hereafter simply "Characterisation"). In this Introduction we present requirements and place the model in the broader context of VO data models. In Section 3 we introduce the concepts (illustrated with some examples) and discuss their interactions. In Section 4 we present a formal UML class model using the concepts defined earlier. XML and VOTABLE serializations are presented in Section 5 and the Appendices give further examples.

\subsection{The purpose of the Characterisation model}

Characterisation is intended to define and organize all the metadata necessary to describe how a dataset occupies multidimensional space, quantitatively and, where relevant, qualitatively. The model focuses on the axes used to delineate this space, including but not limited to Spatial (2D), Spectral and Temporal axes, as well as an axis for the Observable (e.g. flux, number of photons, etc.), or any other physical axes. It should contain, but is not limited to, all relevant metadata generally conveyed by FITS keywords.

Characterisation is applicable to observed or simulated data ${ }^{1}$ but is not designed for catalogues such as lists of derived properties or sources (see Section 2.3).

The model is intended to describe:

- A single observation;

- A data collection;

- The parameter space used by a tool or package accessed via the VO.

The model describes the available data, not its history. For instance, spatial resolution expresses the level of smearing of the true sky brightness distribution in a data set without differentiating between contributions from different atmospheric, instrumental and software processing effects (see Section 2.3).

Characterisation has to satisfy two sets of requirements:

I Data Discovery requirements:

This model prescribes elements for use in requests to databases and services and thus forms a fundamental part of the standards for VO requests. The use of this model should enable a user ${ }^{2}$ to select relevant observations from an archive efficiently. The selection will be

\footnotetext{
${ }^{1}$ Unless otherwise stated, we use the terms "dataset", "observations" etc. to mean any applicable observed or simulated data.

${ }^{2} \mathrm{~A}$ user is either a human or a software agent
} 
based purely on the geometry of the observations, that is, how and how accurately the multidimensional space is covered and sampled.

Discovery may only require a simplified overview (e.g. position, waveband, average spatial resolution). Data providers may opt for the inclusion of data where there is insufficient information to respond to certain parts of a query. Eventually, it should be possible for a client to generate a detailed multidimensional footprint of an observation. For example:

- What observations from a particular archive are likely to have covered a specific VO Event? (Spatial and Temporal Coverage)

- Which CCD frames in a mosaic actually cover the position of a particular galaxy? (detailed Spatial Coverage)

- What observed spectra have a resolution comparable with a given simulated spectrum e.g. matching the Shannon criterion? (Sampling Precision).

II Data Processing/Analysis requirements:

Characterisation should detail the variation of sensitivity on all relevant axes (e.g. variation of sampling or sensitivity across the field of view, detailed bandpass function), in order to provide information to an analysis tool or for reprocessing.

Errors may be provided for any or all axes.

Version 1 will fulfill all Data Discovery requirements, and allow some simple automatic processing such as cross-correlation and data set comparisons. Full implementation of Data Processing/Analysis requirements will only become available in a future version of this model.

\subsection{Scope of the document}

This document defines metadata items and organisation patterns for characterizing data products and their properties in the VO. It identifies some major contexts in which these patterns play a crucial role and it shows how metadata descriptions can be constructed in these contexts, in a form that is adjusted to the requirements for distribution and analysis of astronomical observations. However, the precise application of these patterns in other contexts may be different from these and we do therefore as yet not prescribe the precise syntax of characterization metadata in all contexts. That is left to the controlling documents. 


\subsection{Links to other IVOA modeling efforts}

Characterisation arose out of the "Observation Data Model", a high level description of metadata associated with observed data, described in an IVOA note available at http://www.ivoa.net/Documents/latest/DMObs.html. The connection is summarised in Fig.1. It became obvious that there was an urgent need for a model to characterise the physical properties of data, alongside Provenance, DataCollection, Curation etc. (which provide instrumental, sociological and other information). For example, Provenance will be linked with Characterisation to provide the telescope location (needed for some coordinate transformations), calibration history, etc. Interactions are organised so that each Observation object of the Observation data model will have a Characterisation object encompassing the metadata relative to the physical axes along which the measurements are spanned.

Characterisation complements and extends some of the metadata adopted by the VO Registry (http://www.ivoa.net/Documents/latest/RM.html), providing the finer level of detail needed to describe individual datasets. Concurrently to the Characterisation DM, the Spectrum modeling effort appeared (http://www.ivoa.net/Documents/latest/SpectrumDM.html), focusing on spectral data sets. It partly re-uses the Characterisation metadata tree representation for data spanned along the spectral axis. Data models for Catalogues and Sources are also being developed. Ideally, all these models must be mutually consistent and employ the definitions supplied by the STC DM and/or by basic models describing astronomical quantities as was planned within the Quantity DM. However some overlap and duplication may happen to allow data and service providers to use the parts they need without excessive effort.

\section{Exploring the Characterisation concepts}

\subsection{Overview: a geometric approach}

We introduce the physical axes used to define the N-dimensional space occupied by any data set or required for interpretation. When considering a typical astronomical observation, we have identified various Properties:

- Coverage: describes what direction the telescope was pointing in, at which wavelengths and when; and/or the region covered on each axis. This is described in increasing levels of detail (see Section 3.6.1) by:

- Location

- Bounds

- Support 


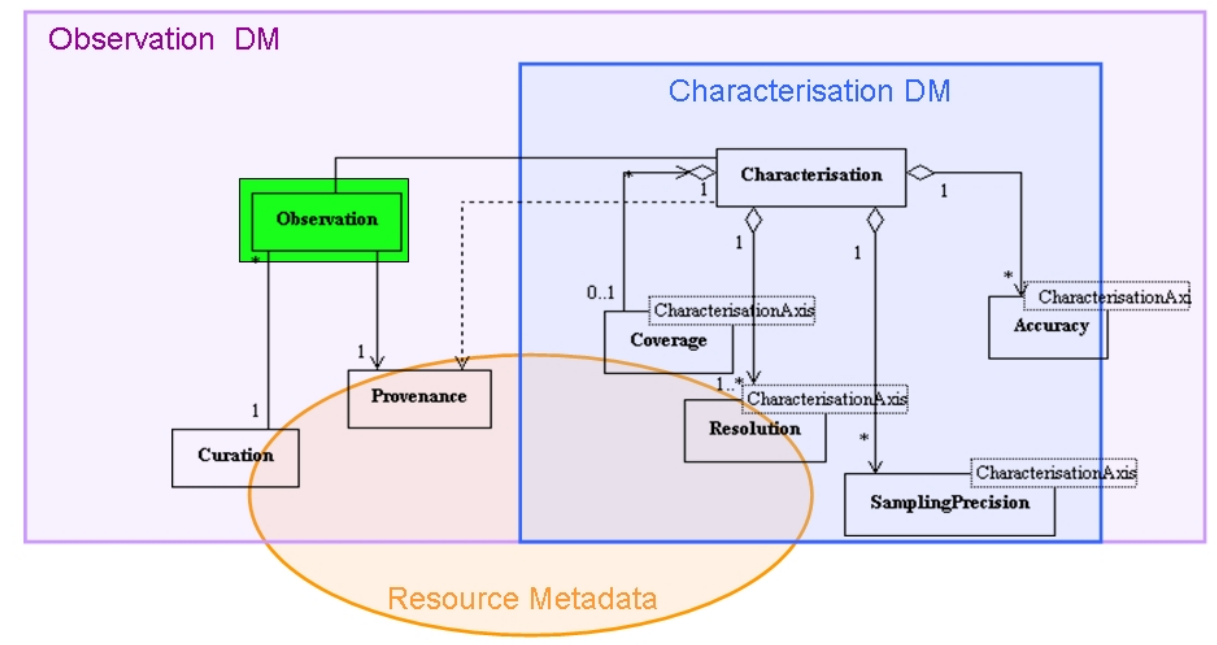

Figure 1: Interaction between the Observation and Characterisation data models: the Characterisation DM focuses on the physical information relative to an Observation, a major class of the Observation Data Model. Characterisation DM has been identified as an important building block within the Observation Data Model, a large scope modeling effort to be completed in the future. Data management aspects such as VO identifier, data format, etc.. are handled in other metadata description like Resource Metadata or parts of the Observation model.

\section{- Sensitivity}

If the data contain many small regions then the Bounds may be qualified by a

\section{- Filling Factor}

(especially if the Support is not precisely defined).

- Sampling Precision: describes the sampling intervals on each axis;

- Resolution: describes the effective physical resolution (e.g. PSF, LSF, etc.).

Each property can be related to one or more physical axes, described in more detail in Section 3.6. For each axis:

- Accuracy: describes the measurement precision, see Section 4.2.2. 


\subsection{Examples of Characterisation}

The tables below illustrate how the spatial, temporal and spectral domains and the observable quantity of some typical data sets can be described, at various levels of complexity, using the properties from Section 3.1. Table 1 shows some of the Characterisation metadata for an X-ray event list. Additional examples are presented in Appendix $\mathrm{C}$ : Table 2 for a 2-D image, Table 3 for a 1D spectrum, Table 4 for an IFU Dataset, Table 5 for a radio interferometry image service and Table 6 for simulated data.

In some of these examples, some concepts are interdependent, discussed further in Section 3.4.1. All these concepts can be applied to any data set but some elements may not have defined values, or the origin may be arbitrary, for example the spatial location of a generic simulated galaxy cluster (Table 6).

\subsection{Structure and development strategy}

Characterisation provides a framework to present the metadata necessary to specify a dataset in a standard format and to make any interrelationships explicit. The description can be presented from the perspective of the Properties or the Axes in a succession of progressively more detailed description layers. This will allow evolution of the model in three independent directions: new properties may be added as well as new axes, and if necessary new levels of description may be considered without breaking the overall structure.

\subsection{The Axis point of view}

\subsubsection{Axes and their attributes}

The physical dimensions of the data are described by axes such as: Spatial, Spectral, Time, Velocity, Visibility, Polarisation, Observable. We recommend that data providers use these axes names but this is not compulsory (e.g. FITS names can be used). The data provider will be required to supply a UCD for each axis, as well as the units. This would help to disentangle ambiguous or unprecise metadata for data retrieval or recognition by standard software. There is no limit on the number of axes present and they may be dependent or overlapping (e.g. one frequency axis and two velocity axes, representing the velocities of two separate molecules with transitions at similar frequencies).

Some axes may not even be explicit in the data, but are implicit, present only as a header keyword or elsewhere. For example, a simple 2D sky image 


\begin{tabular}{|c|c|c|c|c|}
\hline $\begin{array}{l}\text { AXES } \\
\text { PROPERTIES }\end{array}$ & SPATIAL & TEMPORAL & SPECTRAL & $\begin{array}{l}\text { OBSERVABLE } \\
\text { E.G. FLUX }\end{array}$ \\
\hline \multicolumn{5}{|l|}{ Coverage } \\
\hline Location & $\begin{array}{l}\text { Centra } \\
\text { position }\end{array}$ & Mid- Time & $\begin{array}{l}\text { Central } \\
\text { energy }\end{array}$ & Average flux \\
\hline Bounds & $\begin{array}{l}\text { RA,Dec } \\
{[\text { min,max] or }} \\
\text { Bounding box } \\
\text { [center, size] }\end{array}$ & $\begin{array}{l}\text { Start/stop } \\
\text { time }\end{array}$ & $\begin{array}{l}\text { Energy } \\
{[\min , \max ]}\end{array}$ & $\begin{array}{l}\text { min:Probability } \\
\text { above } \\
\text { background } \\
\text { max: Pileup }\end{array}$ \\
\hline Support & $\begin{array}{l}\text { FOV as accurate } \\
\text { array of } \\
\text { polygons }\end{array}$ & $\begin{array}{l}\text { Time } \\
\text { intervals } \\
\text { (array) }\end{array}$ & $\begin{array}{l}\text { Energy filter } \\
\text { intervals } \\
\text { (array) }\end{array}$ & \\
\hline Sensitivity & $\begin{array}{l}\text { Quantum } \\
\text { efficiency }(\mathrm{x}, \mathrm{y}) \text {; } \\
\text { vignetting }\end{array}$ & & $\begin{array}{l}\text { ARF (effective } \\
\text { area)as fn } \\
\text { of energy }\end{array}$ & $\begin{array}{l}\text { Out-of-time } \\
\text { events } \\
\text { (saturation); } \\
\text { wings of PSF }\end{array}$ \\
\hline $\begin{array}{l}\text { Filling } \\
\text { factor }\end{array}$ & $\begin{array}{l}\text { Good pixel } \\
\text { fraction }\end{array}$ & $\begin{array}{l}\text { Live time } \\
\text { fraction }\end{array}$ & $\begin{array}{l}\text { not } \\
\text { used }\end{array}$ & \\
\hline Resolution & $\begin{array}{l}\text { PSF (x,y) } \\
\text { or its FWHM }\end{array}$ & $\begin{array}{l}\text { Time } \\
\text { resolution }\end{array}$ & $\begin{array}{l}\text { RMF (spectral } \\
\text { redist. matrix) }\end{array}$ & SNR \\
\hline $\begin{array}{l}\text { Sampling } \\
\text { Precision }\end{array}$ & $\begin{array}{l}\text { Pixel scale } \\
(x, y)\end{array}$ & $\begin{array}{l}\text { Frame } \\
\text { time }\end{array}$ & $\begin{array}{l}\text { PI bin } \\
\text { width }\end{array}$ & $\begin{array}{l}\text { ADU } \\
\text { quantization }\end{array}$ \\
\hline
\end{tabular}

Table 1: Property versus Axis description of metadata describing an $\boldsymbol{X}$-ray CCD Event List. This also characterises the potential images and other products which can be derived. During exposure, the instrument moves with respect to the sky, so, for example, the sensitivity is a function of the support on the first three axes. 
usually has celestial coordinate axes, but the time and spectral axes may not be present in the main data array although the observation was made using a finite integration time and wavelength band (a single sample on each of the temporal and spectral axes). These implicit axes may be represented in Coverage to provide their location an/or bounds, or even, for purposes such as color corrections, their sensitivity as a function of the coordinate within the bounds.

\subsubsection{Axes flags}

Axes flags (Section 4.2.1) are used to indicate Boolean and other qualifying properties. These include whether the axis represents a dependent variable (e.g. the Observable), the calibration status and whether the data are undersampled.

\subsection{Accuracy}

Accuracy characterises any uncertainties associated with each axis (Section 4.2.2) - astrometric uncertainties are attached to the Spatial axis, photometric to the Observable etc. Note that this is a level of detail distinct from the assessment of the overall accuracy of data provided by the Registry metadata.

\subsection{The Property point of view}

The main properties needed for data description and retrieval are categorized under Coverage, Resolution, and SamplingPrecision, introduced in Section 3.1 .

The values of the properties characterising an Observation may be derived from instrumental properties given in Provenance or from other Characterisation features. For example, high energy missions move the telescope during the observation (Table 1), leading to a time-variable mapping from detector to celestial coordinates (the 'aspect solution'), giving a spatially variable effective exposure time derived from the temporal bounds multiplied by the filling factor, or the sum of all the support intervals weighted by sensitivity, or derived from the sampling precision and period within the bounds. The sensitivity across the spectral band may be a function of spectral position (ARF). Such dependencies should be restricted to areas of significance to users, such as the Sensitivity class. At present, a single value, or the extrema, can be given for each element; more complex formulae will be available in a future version of Characterisation. 


\subsubsection{Coverage}

Coverage has several levels of depth, providing a range of detail to meet the needs of any user/developer, illustrated in Fig. 2. The simplest approximation to a spatial field of view presumes that a sharp-edged region of the celestial sphere has $100 \%$ sensitivity inside and $0 \%$ outside. In reality the transition is fuzzy and the region may be irregular and contain gaps. For example, some applications only need to know what range of coordinate axes values might contain data; others need to know the variation in (flux) sensitivity as a function of position on an axis. Coverage provides answers to these questions at different levels of precision, with the idea that software implementations will be able to convert between the levels.
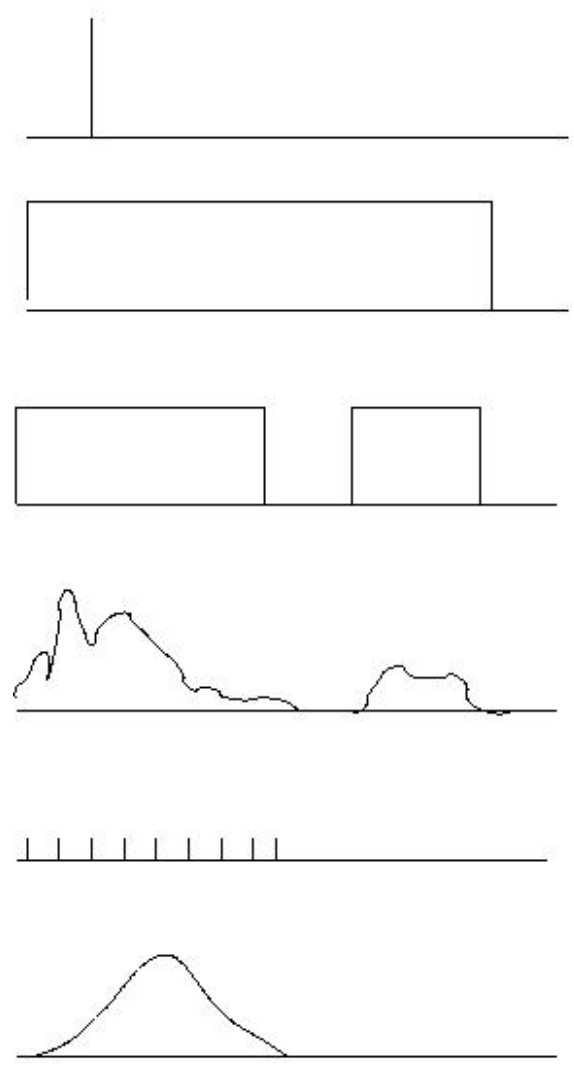

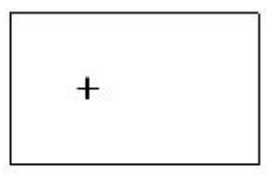

Bounds

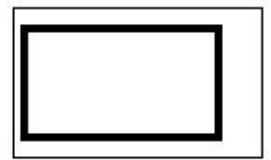

Support

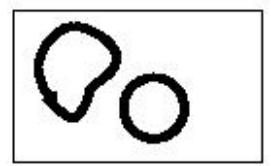

Sensitivity

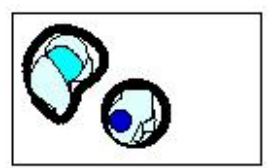

Precision

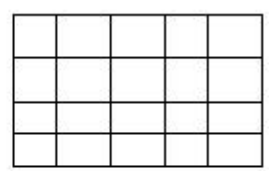

Resolution

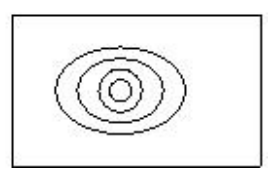

Figure 2: Illustration of the different levels of description. left: for a 1dimensional signal, right: for a $2 D$ signal.

Coverage is described by four layers which give a hierarchical view of increasing detail:

1. Location: The simplest Coverage element is the Location of a point 
in N-dimensional parameter space, such as an image described by a single value each of RA, Dec, wavelength and time. These are fiducial values representative of the data. A precise definition (mean, weighted median, etc.) is not required, but Location can serve as a reference value or origin of coordinates in frames with no absolute position (e.g. Table 6).

2. Bounds: The next level of description is the SensitivityBounds, i.e. a single range in each parameter providing the lower and higher limits of an N-dimensional "box". The scalar intervals between the limits (the sizes and centres of each box-side) should also be available if required. The Bounds are guaranteed to enclose all valid data but there may be excluded edge regions for which there is no valid data, such as (on the wavelength axis) the 'red leak' end of a spectral filter. These provisions satisfy the intent of typical data discovery queries.

3. Support: Mathematically, the support of a function is the subset of its domain where the function is non-zero. Here, Support describes quantitatively the subsets of space, time, frequency and other domains, onto which the observable is mapped, where there are valid data (according to some specified quality criterion). Support may include one or many ranges on each axis (e.g. Table 4).

4. Sensitivity: Sensitivity, (unlike the previous 'on/off' properties), provides numerical values indicating the variation of the response function on each of the axes, such as the relative cell-to-cell sensitivity in the data. This includes filter transmission curves, flat fields, sensitivity maps, etc. The final limits on Sensitivity are determined by the bounds of the Observable; for example, the minimum and maximum given by a single count and by the saturation level for some types of detector.

The Bounds may also contain the

- Filling Factor sub-level, which gives the useful fraction of Bounds on any axis. It may not be appropriate to detail multiple small interruptions to data (for example detectors requiring dead time between each sample) if it is conventional for analysis systems to solve the problem using a statistical correction based on the Filling Factor. Very regular filling may also be described by Sampling (see below). Even if Support provides a complete description, the Filling Factor may be used to rank the suitability of data during discovery.

A method should be provided to derive the Filling Factor from the Sampling Extent and Sample Precision (Section 3.6.2) if these are given, but if all three values are entered separately there needs to be a means of checking for consistency. 


\subsubsection{Resolution and Sampling Precision}

Resolution is often a smoothly decaying (e.g. Gaussian) function but the data product is subject to further discrete Sampling, e.g. CCD pixels, Table 2. Resolution may, however, be a top hat function determined by the Sampling interval - e.g. the temporal resolution of an image made from a single integration. We maintain a distinction between the concepts to facilitate different requirements in data processing, whether during data discovery services which allow resampling or flexible resolution (Table 5), or during post-discovery processing (Table 4).

- Resolution Resolution is usually the minimum independent interval of measurement on any axis. Mathematically, if the physical attributes (e.g. position, time, energy) of the incident photons, or other observable, are $\mathbf{x}$ (e.g. $x_{0}=$ energy, $x_{1}=\mathrm{RA}, x_{2}=$ Dec, $x_{3}=$ time, etc.), and the measured attributes are $\mathbf{y}$ (e.g. $y_{1}=$ spectral channel, $y_{2}, y_{3}$ $=$ pixel position, $y_{4}=$ time bin) then given a flux of photons $S(\mathbf{x})$ the detected number of photons is

$$
N\left(y_{1}, y_{2}, \ldots\right)=N(\mathbf{y})=\int \mathbf{S}(\mathbf{x}) \mathbf{A}(\mathbf{x}) \mathbf{R}(\mathbf{x}, \mathbf{y}) \mathbf{d} \mathbf{x}
$$

where $\mathrm{A}$ is the probability that a photon is detected at all (the quantum efficiency) and $R\left(x_{1}, x_{2}, \ldots, y_{1}, y_{2}, \ldots\right)$ is the smearing of measured values (PSF, line spread function, etc.).

In the most detailed case, $\mathbf{R}(\mathbf{x}, \mathbf{y})$ may be a complicated function, such as a PSF which varies as a function of detector position and energy. The first level of simplification is to specify a single function which applies to the whole observation - e.g. a single PSF. This function may either be provided as a parameterized predefined function (e.g Gaussian) or as an array. The concept of Resolution Bounds provides the extreme values of resolution (see Table 5)

The final level of simplification is to give a single number characterising the resolution, such as the the standard deviation of a Gaussian PSF.

- Sampling

Sampling (or pixelization or precision or quantization) describes the truncation of data values as part of the data acquisition or data processing. If sampling is non-linear, simplification may be necessary, by giving limiting values or a single 'characteristic sampling precision'. The Sampling Period gives the sample separation and the Sample Extent shows the deviation from the pure "Dirac comb" case. The Nyquist parameter - the ratio between the resolution FWHM and the Sampling 
period - will also be provided by a method. The Sampling flags (Section 4.2.1) provide a simple guide as to whether these properties are significant.

\subsection{Presentation of layered information}

The layered structure allows tasks to retrieve only the metadata which is actually required. The lower levels can be very detailed, for example the variation in Sensitivity to the Observable(s) along the spatial, spectral and other axes, or the variation of the resolution within the field of view. This could take various forms:

- A simple value or range

- An analytic function of other property values

- A variance map for $2 \mathrm{D}$ data

- A look-up table for the bandpass correction to 1D spectral data

The more complex properties may be provided using pointers to ancillary data with the same types of axes and dimensions as the observation itself, e.g. a weight map packaged with a 2D image; this capability exists in the first version of this model. The provision of "attribute formulae" or attributes pointing to functional descriptions, such as the aspect solution for an X-ray observation, is left for the future development of Characterisation; a first step may be to decompose a complex coupled description into non-coupled expressions. Where it is possible to provide separate values for interdependent elements (see also the end of Section 3.6.1), there must be a validation method to avoid contradictions.

A later version of the model will also allow links to other aspects of the Observation model, external calibration and documentation. Advanced VO tools could use such metadata to recalibrate data on demand. Characterization is used to describe potential as well as static data products (e.g. Tables 1 and 5). It could therefore also provide pointers to Registry entries indexing tools and services that could be launched on the fly for extracting images etc. from event or visibility data or atlas cut-outs.

\section{The Model}

\subsection{The role and structure of the Model}

We use UML diagrams to describe the organisation of Characterisation metadata following the Properties/Axis/Levels perspective. The model offers 
different views of the characterisation concepts. Figure 3 shows the relationships between the main concepts. The AxisType box attached to each property class represents the axes along which the property (e.g. Resolution) is assessed; for example, there can be one Resolution class for each relevant axis. Fig 4 illustrates how the properties of the data are gathered under the Characterisation container class. The Coverage class is shown with the four increasingly detailed properties introduced in Section 3.6; such a Characterisation tree is available for each type of axis.

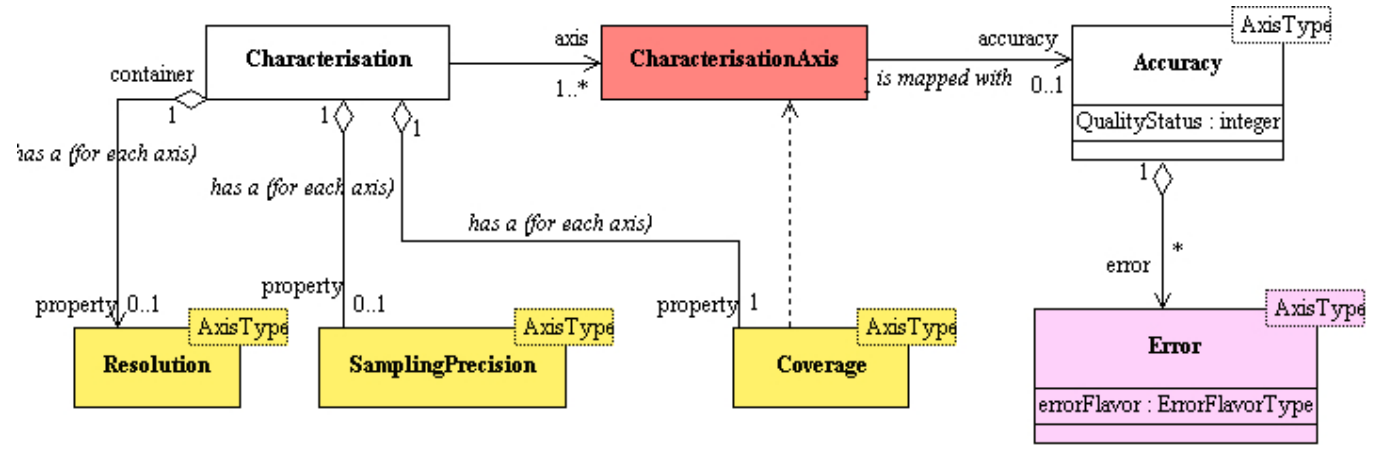

Figure 3: This UML class diagram emphasises the Property/Axis perspective. The Characterisation class is a container that gathers the properties for each axis. The axis is described by the CharacterisationAxis class. All relevant axes for one observation/dataset are linked to the Characterisation class. The Axis Type template parameter for each Property allows to link properties to the corresponding Axis. The Accuracy class, linked to the CharacterisationAxis class, gathers different types of Error descriptions (systematic, statistical) as well as quality flags.

\subsection{Axis description}

All the information related to an axis is gathered within the CharacterisationAxis class. This can have common "factorised" attributes applicable to the property layers on that axis (Section 3.1). It contains the name of the axis, units, UCD as well as a holder for the STC coordinate frame (see Section 4.4) which also provides the base class for the observatory location (Observation - Provenance model).

If a deep level (higher number, Section 3.6) object, e.g. Sensitivity, needs to have its own axis description, this can be defined locally, overriding the factorised top level CharacterisationAxis object. The redefinition can be 


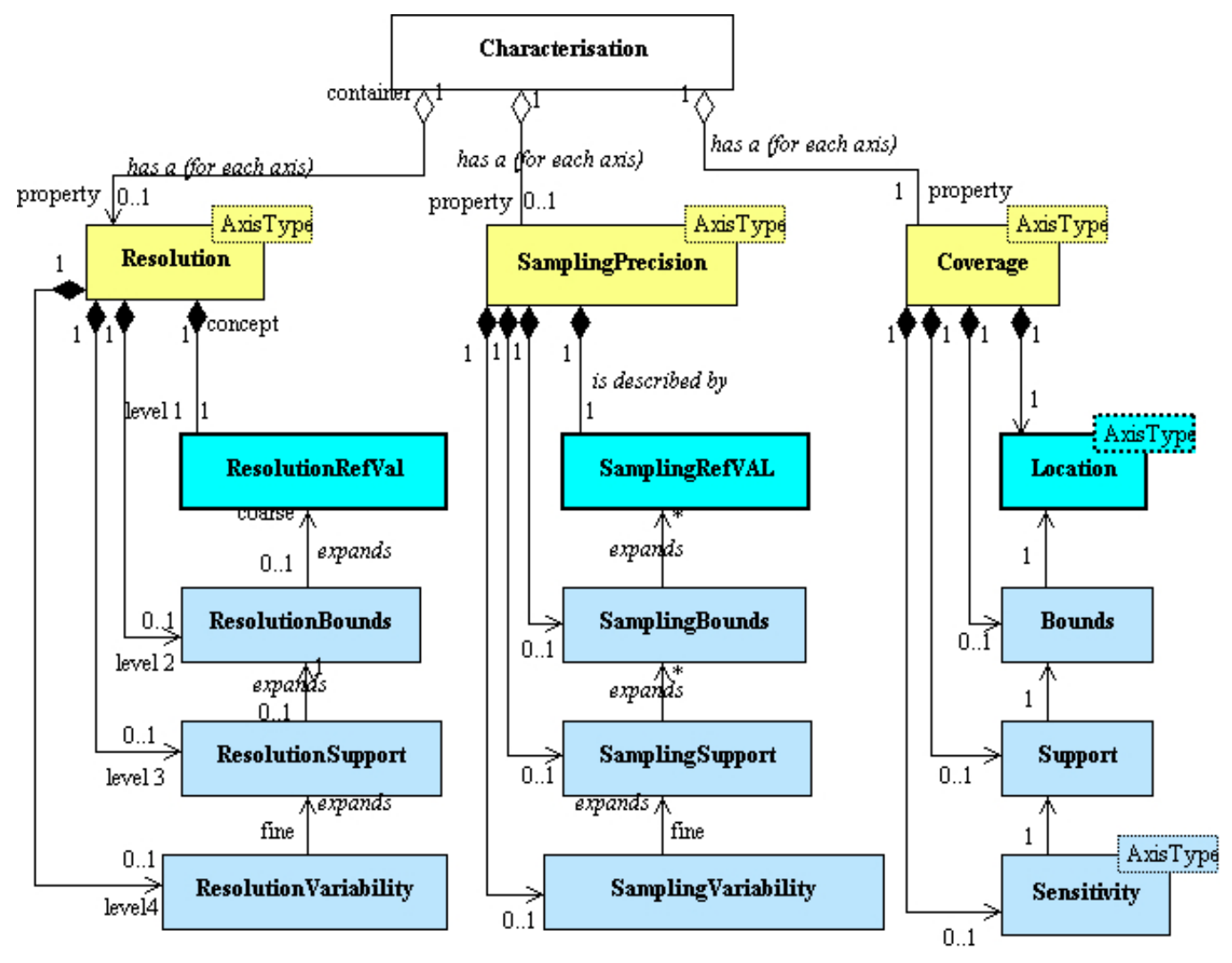

Figure 4: The layered structure of Characterisation: This diagram synthesises the Property/Axis/Layer approach. The concepts are represented in yellow. The coarse description is designed by the first level (blue boxes), while the pale blue ones represent the complementary metadata. The Bounds, Support and Sensitivity classes are nested levels of detail to add knowledge about the Coverage of an Observation. Symmetrically, Resolution and Sampling may also have the 4-level structure of description. The complete Characterisation for one observation is obtained by filling the tree for each relevant axis: spatial, spectral, temporal, etc. 
partial, e.g. a change of unit or a change of spatial orientation requiring a new CoordSystem element.

\subsubsection{Flags and other qualifying information}

Other elements in the CharacterisationAxis class include the number of bins present on this axis, and flags to indicate the calibration status, independency and sampling properties of the axis, as described in Section 3.4.2

4.2.1.1 Independent or dependent status Axes may include both 'independent' variables (which may have associated errors) and the "Observable" axis or axes which represent phenomena measured along some other axes. For instance, in a 3D datacube of the sky, the Spatial axis is an independent axis (flag TRUE), as is the (implicit) Spectral axis, but the Flux axis is dependent (flag FALSE), and the velocity axis is dependent on the frequency axis.

4.2.1.2 Calibration status The CharacterisationAxis object in the Characterisation model provides a calibration status flag for each axis, so that a user can insist on calibrated data only where necessary. The CalibrationStatus is given separately for each type of characterisation axis and can be

- UNCALIBRATED: not in units which can be directly compared with other data (but often still useful, for example the presence of spectral lines at known wavelengths can give a redshift regardless of absolute flux densities).

- CALIBRATED: in reliable physical units or other accepted units such as magnitudes. ${ }^{3}$

- RELATIVE: calibrated to within a constant (additive or multiplicative) factor which is not precisely known, such as arising from uncertainty in the flux density of a reference source.

- NORMALIZED: dimensionless data, divided by another data set (or a local extremum).

The calibration process itself is described elsewhere in the Observation Data Model (Section 2.3).

\footnotetext{
${ }^{3}$ In such cases the coarser levels of description should also be given in physical units and the need for a tool such as a look-up table of zeropoints etc. and conversion algorithms has been identified.
} 


\subsubsection{Sampling status}

- Undersampling: TRUE if the sampling precision period is coarse compared to the resolution and the precision of a single data value is limited by the sampling; FALSE if the sampling precision period is small compared to the resolution and precision is limited by the resolution

- Regular sampling: TRUE if the pixellation or binning is close to linear with respect to the axis world coordinate (so that an accurate position can be obtained by counting samples from a Bound); FALSE if this would introduce an error significant with respect to other uncertainties.

- The total number of samples along each axis may be given, normally used for multiple regular sampling.

\subsubsection{Errors in Characterisation: the Accuracy class}

The values along Coordinate axes and measurements of Observables may all suffer from systematic and statistical uncertainties. Errors may be in the units of the axis or may be represented by quality flags. These Error classes are gathered in an Accuracy object (linked to the CharacterisationAxis object, see Fig. 5, and STC data model elements, see Section 4.4)). Accuracy supports multiple levels of description, analogous to Coverage. The uncertainty in the position or measurement on any axis can be described by a typical value, by the bounds on a range of errors, and/or by very detailed error values for each sampling element (e.g. pixel). ${ }^{4}$ A pointer may be provided to error maps packaged with the data, as described for the more detailed levels of Coverage.

\subsection{Navigation in the model: by axis or by properties?}

The structure of Characterisation is clearly hierarchical with the characterisation class as the root element. The model can be serialised using two alternative sets of primary elements:

- Properties, with the corresponding classes for each axis attached; used, for example, to represent data where the axes values are interdependent (e.g. Table 1);

- Axes, factorising each description into the multi-layer property levels; this provides more compact XML.

\footnotetext{
${ }^{4}$ Measurement errors are distinct from any 'fuzziness' in the values provided by the coarsest levels of Characterisation, e.g. Location may be an arbitrary approximation (Section 3.6.1), but that kind of uncertainty is catered for by going to deeper levels of Characterisation, and by the concept of Region of Regard in the Registry Resource model.
} 


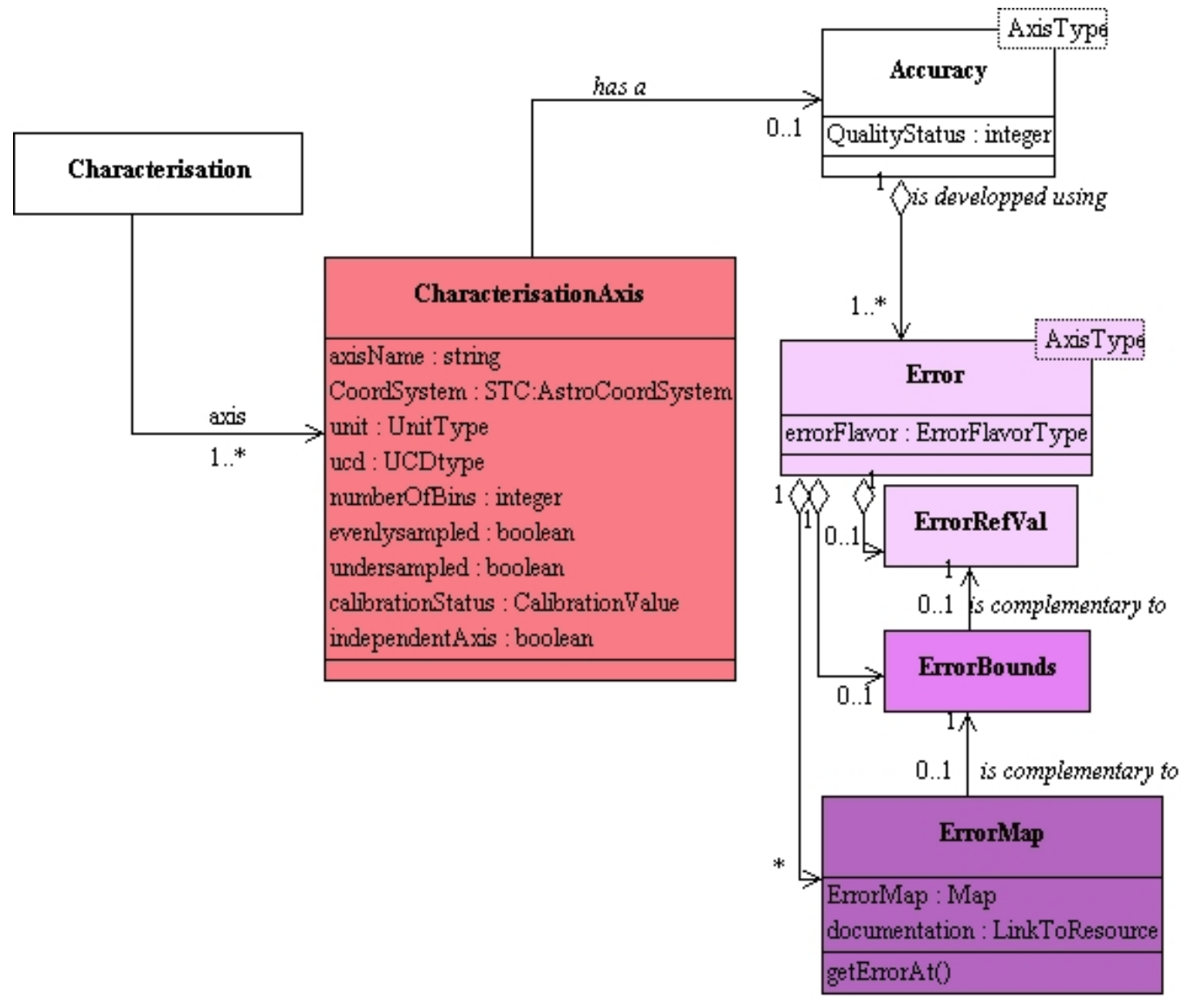

Figure 5: This class diagram illustrates the CharacterisationAxis class and its relationship with the Accuracy class, which encompasses various types of errors such as systematic or statistical. 
Either structure could be applied to the examples tabulated in Section 3.2. This UML model could be used to build two different XML schemas, giving access primarily by property or by axis. Here, we present the "Axis First" serialisation only; the "Property First" serialisation will be presented in the next version of this model.

\subsection{Implementing the model using STC elements}

STC, the metadata scheme for Space-Time Coordinates (see http://www.ivoa.net/Documents/latest/STC.html) encompasses the description of most of the Characterisation axes examples in Section 3.4.1 with the exception of Observable. Sensitivity is the only Property not present in STC. However, the full STC structure cannot simply be reused, as it does not have the flexibility needed to deliver the alternative schemata for both multilayered views presented in Sections 4.1 and 4.3. We do use STC intermediate level objects as building blocks for the Characterisation model.

The STC:AstroCoordSystem object is needed as a reference to define the Coverage axes. STC substructures may be reused in the following way:

- Location implements STC:AstroCoords

- Bounds encapsulates STC basic types, some STC:Interval elements and STC:Coords into a structure similar to STC:AstroCoordArea.

- Support uses STC:AstroCoordArea

- Resolution ResolutionRefval can be implemented via adhoc types using STC:CResolution elements

- SamplingPeriod and SampleExtent encapsulate CPixSize elements from STC.

This is represented for the spatial axis using implementation links in the UML diagram in Fig.6.

In simple cases data handlers will probably reuse predefined elements included from an external STC library. For example, CharacterisationAxis includes the STC elements for CoordSys and the (possibly variable) spacetime coordinates of the ObservatoryLocation ${ }^{5}$ or of the origin of coordinates (e.g. for barycenter-corrected data).

Many parameters (i.e. most numerical-valued elements at a finer level than Location) are customarily expressed either as maximum and minimum values or as a centre and scalar range (or both). In some cases an array of such values is needed, e.g. 2 dimensions on the spatial axis in most but

\footnotetext{
${ }^{5}$ This should, where necessary, be consistent with the Provenance section of the Observation model (Section 2.3).
} 


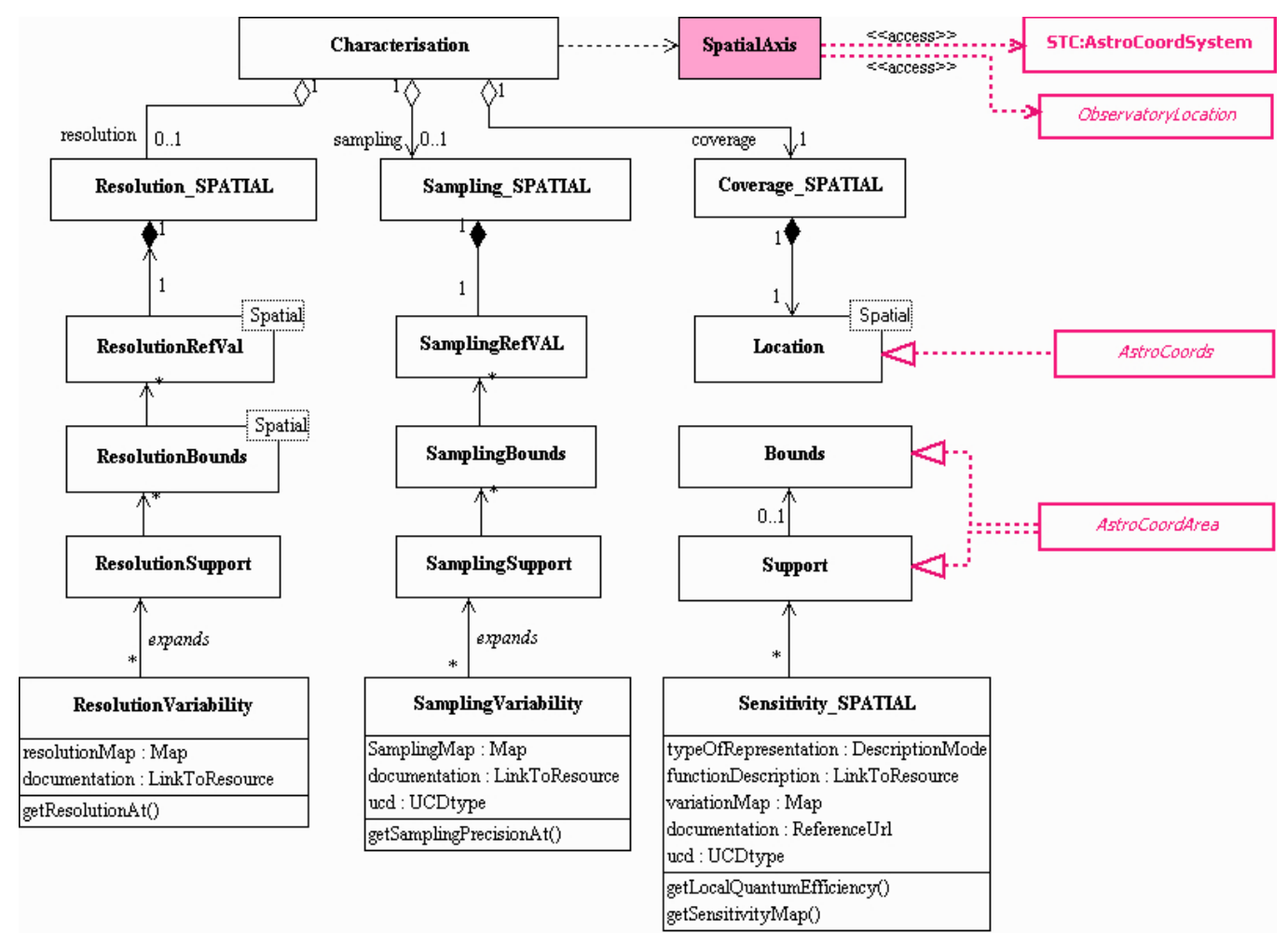

Figure 6: Expressing the spatial properties as a subtree of Characterisation . Here is an example of how STC components (in pink italics) may be used to implement the different levels of the Coverage description. The Location element uses a STC:AstroCoords. Bounds encapsulates STC basic structures like STC:Interval elements and STC:Coords in a structure similar to STC:AstroCoordArea. 
not all cases; upper and lower bounds to (separately) the major and minor axes of Resolution in Table 5; higher dimensionality is possible such as the inclusion of beam position angle in this Resolution example.

The Resolution and Pixel-Size concepts are represented in STC at a deep level inside the Coordinates class (together with the Name/Value/Error in the Coordinate object). This allows any coordinate to be expressed to the appropriate degree of numerical precision. Characterisation needs to allow selection of metadata by resolution, which therefore must be accessible at the upper level of description and is coded as a Property along one CharacterisationAxis, as well as SamplingPrecision.

Since the space, time and spectral axes are particularly important for astronomy, we recommend that implementations include a method to return an STC::AstroCoordSys object, which will only succeed if a complete and consistent space-time-spectral description is present. This may be nominal or arbitrary for some axes e.g. for simulated data.

\section{$5 \quad X M L$ Serialization}

\subsection{XML schema (Axis First)}

\subsubsection{Design of the schema}

Due to the hierarchical nature of the Model, the XML serialization of Characterisation is based here on a single tree. The appropriate elements are taken from STC as described in Section 4.4. The root element called "Characterisation" is the aggregation of a set of CharacterisationAxis elements ${ }^{6}$ for each of the axes. The CharacterisationAxis element contains all axis information like an obvious label ("spatial", "temporal"), coordinate system, units, etc. Coverage implements different elements according to the four levels of description detailed in Section 3.6.1. Lower levels of these properties along one particular CharacterisationAxis may reuse the axis parameters defined into the top-level objects for that axis or redefine their own axis parameters(units, coordsystem, ...) locally, as described in Section 4.2.

A full XML serialisation is provided, as an XML schema, for simple observations, at the following site:

http://www. ivoa.net/xml/Characterisation/v1.11

An XML instance document MPFS-v1.11.xml describing an IFU dataset characterisation is available at http://www.ivoa.net/internal/IVOA/CharacterisationDataModel/.

\footnotetext{
${ }^{6}$ These elements are containers gathering the result of the dynamic grouping of properties for a given characterization axis
} 
Most of the usual (spatial, time, spectral, velocity) coordinates information re-uses the STC coordinates definitions and structures. For simple values, the present version of the schema makes use of STC fine grain structures like CPixsize or CError. Such terminal leaves elements in the Characterisation XML tree could be adapted and re-use simple existing structures (value, unit, semantic tag, coding format) when available in a standard.

\subsubsection{Building blocks of the schemata}

In order to illustrate how the XML schemata is derived from the UML Model, building blocks of the Schemata, corresponding to some main classes of the UML diagram are shown here.

The principle is to map the main classes in XML elements, building up a hierarchy from the most englobing concept down to more specific ones. Aggregated classes are easily translated as aggregated subelements. The attributes of an UML class are also coded as sublevel elements.

The translation from UML to XML used in this serialisation applies rules and elaborates specific techniques very similar to the work of Carlson (Modeling XML applications with UML, Addison-Wesley, 2001). The examples shown here are 'handmade' translations of the UML model. Automated translation will be discussed in the next version of Characterisation. The derivation of the XML from the UML model is expressed in the graphical views of the XML schema in Figs. 7, 8, 10, 11 and 12. 


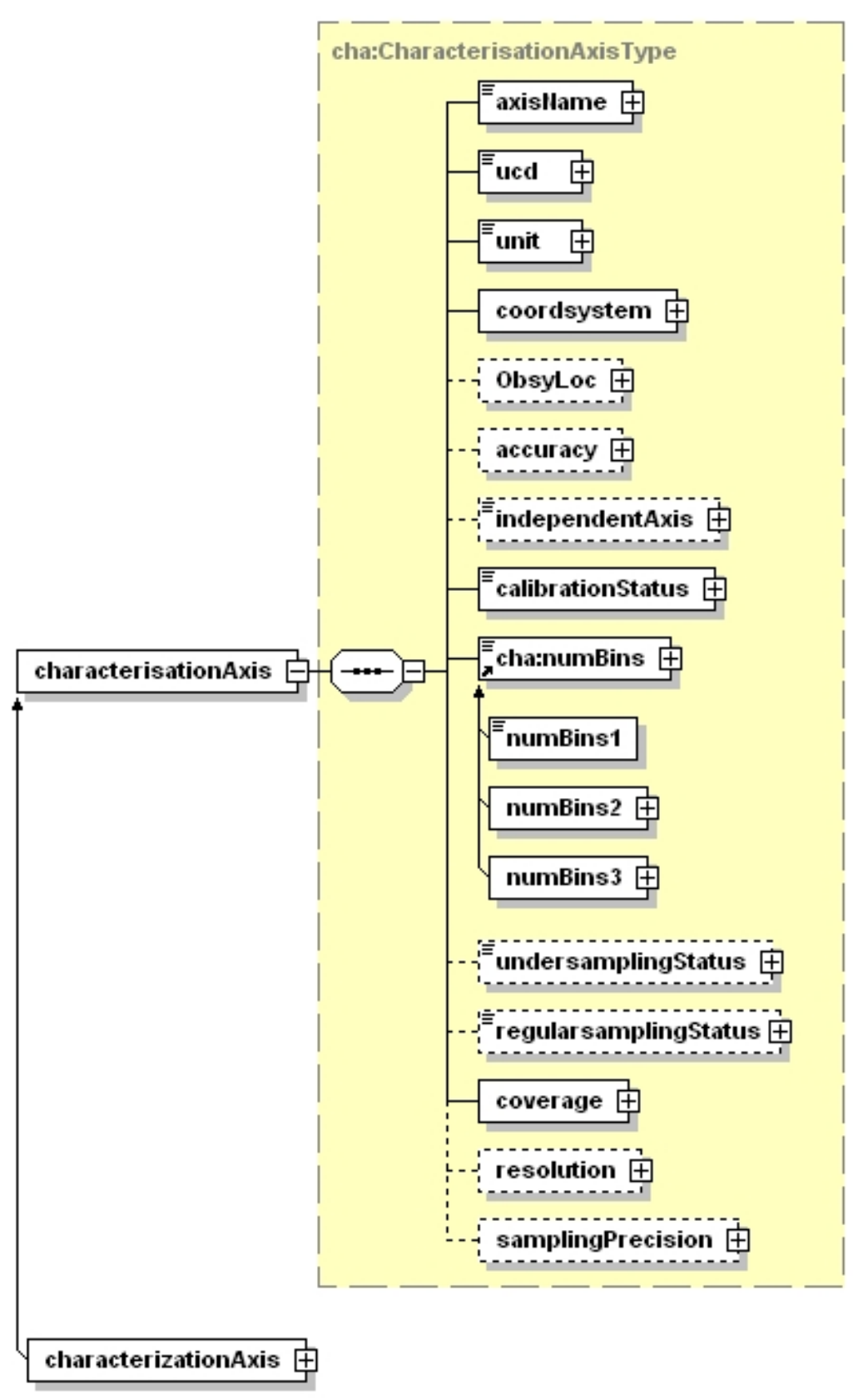

Figure 7: The CharacterisationAxis element is built up following the corresponding UML class with coordsystem and ObsyLoc items reusing STC elements. The small arrow on cha:numbins represents a substitution group head element in $X M L$. This allows to plug various constructs of this element (e.g. for 1D, 2D, 3D) that play the same role in the XML tree. 


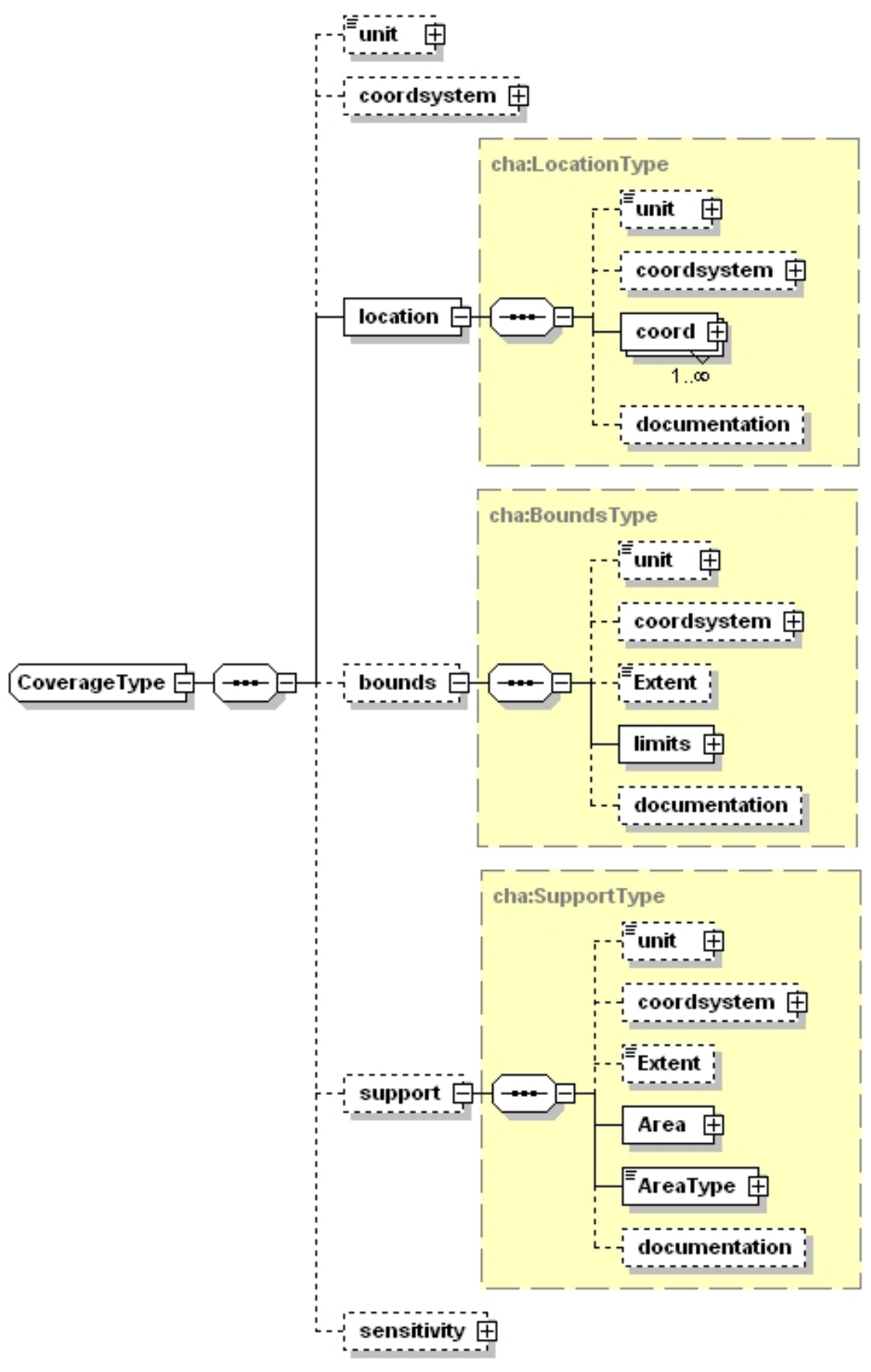

Figure 8: The coordsystem and unit items can be factorised at the top of the Coverage structure, but may be redefined at each level when necessary. Bounds are expressed using a limits element which is developped on a general bounding box type: CharCoordArea. AreaType is a string describing the kind of region used: Circle, Polygon etc. 


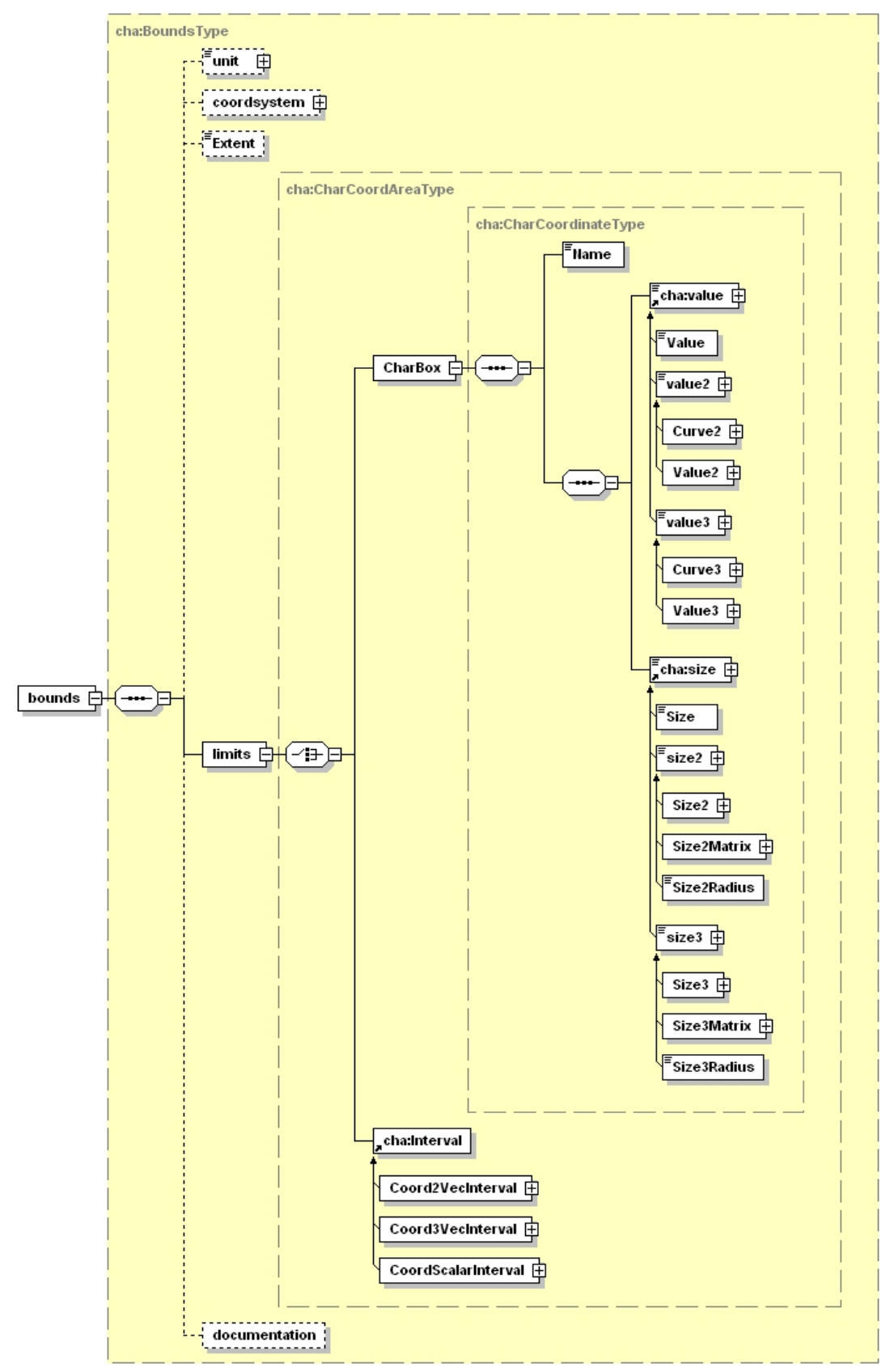

Figure 9: Representing limits: The two expressions allowed for a bounding box are expressed using either a STC:CoordInterval embedded in a locally defined type cha:Interval or built on another type: CharBox representing a generic centered box in $\mathrm{N}$-dimensions. 


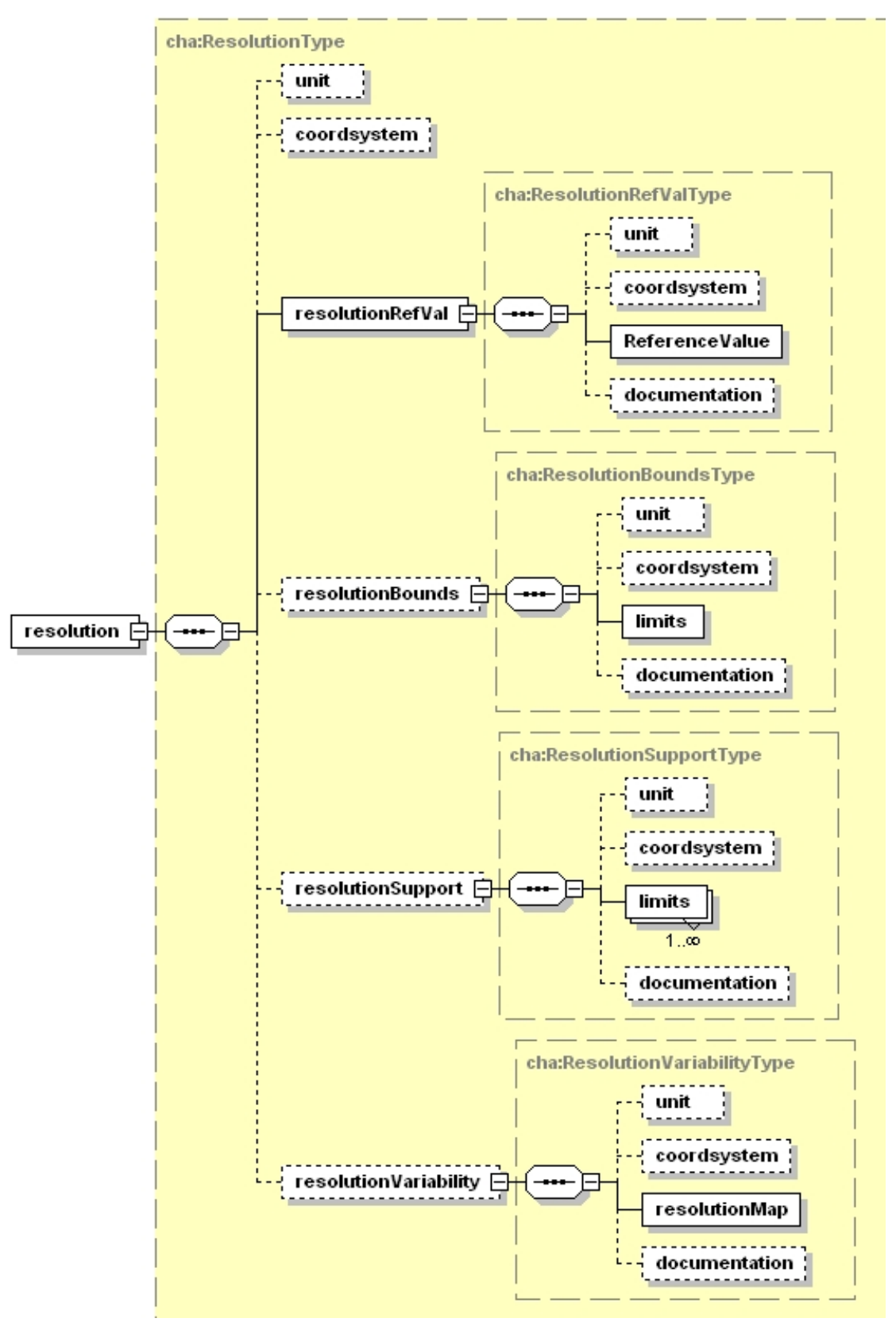

Figure 10: This graphical view was generated with XMLSPY from the resolution element of the schema. As designed in the UML class, the resolution item may contain 4 possible subelements. The RefVal element should be present but is not mandatory: some observations may have unknown resolution. 


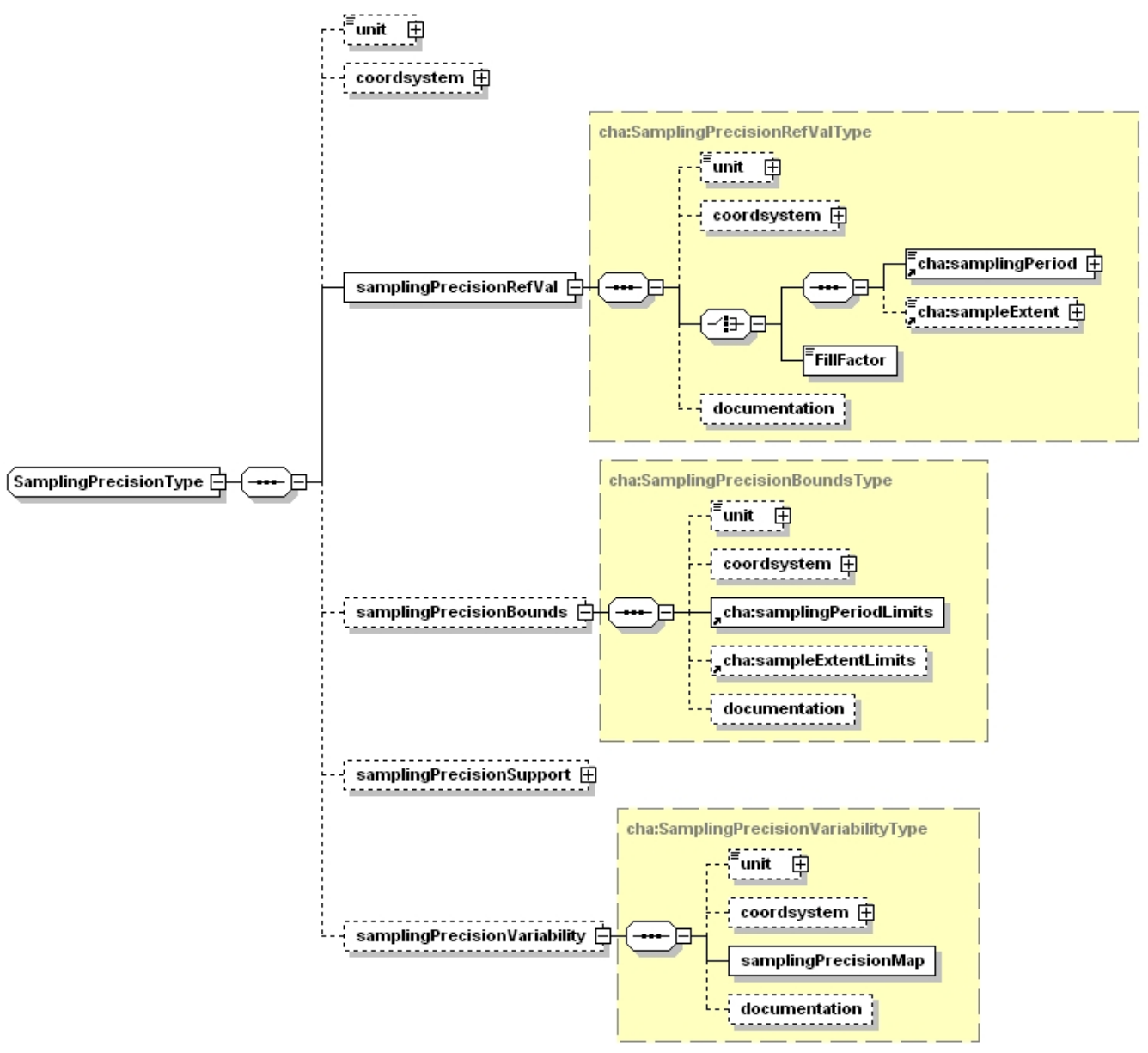

Figure 11: The samplingPrecision item contains 4 possible subelements. One among SamplingPrecisionRefVal and SamplingPrecisionBounds should be present when possible but this is not explicitly described by the XML syntax. 


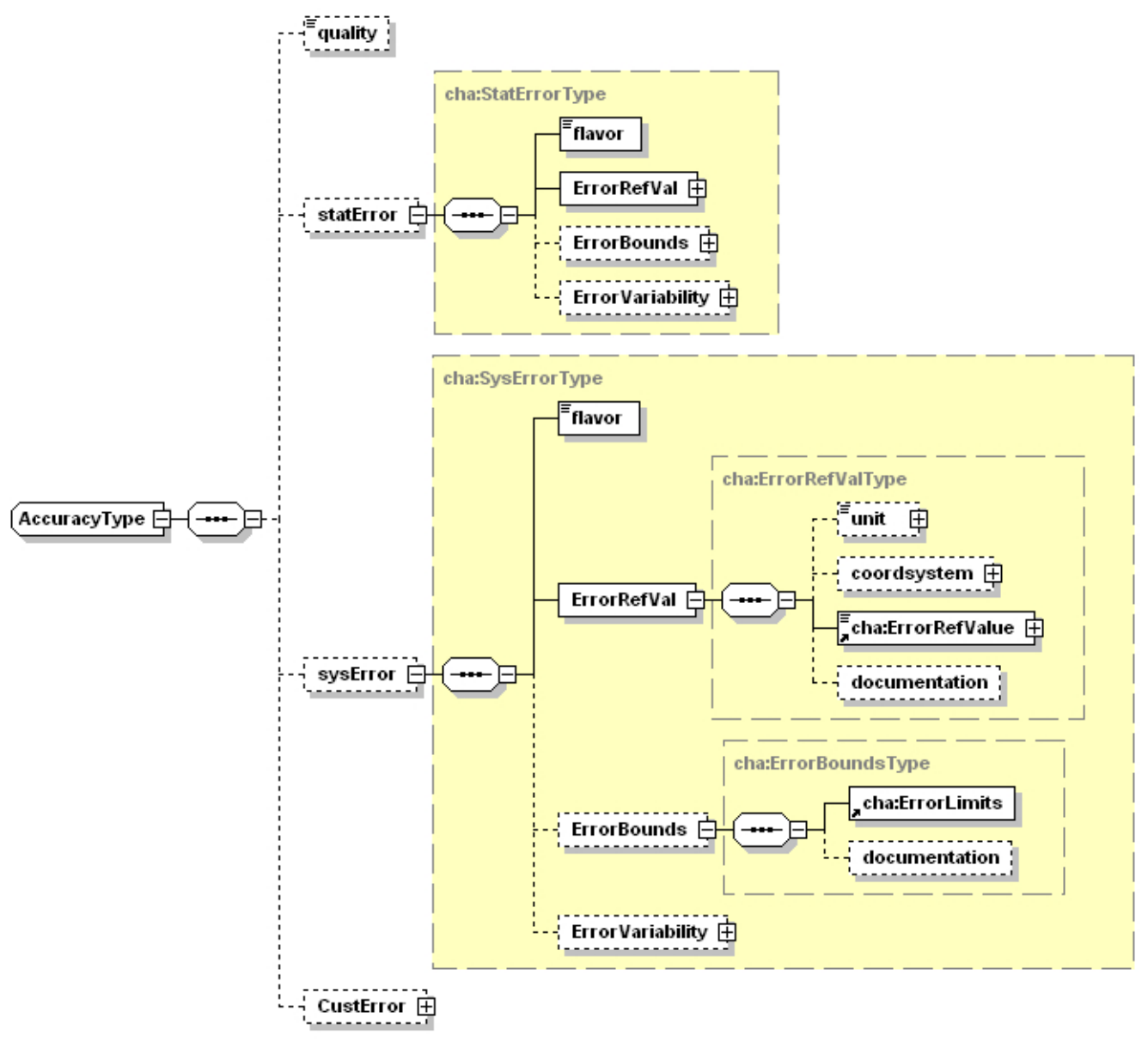

Figure 12: The accuracy element relies on Errors along the axes and is built up on $S T C$ elements. 


\subsection{Utypes generation: select one ordering strategy}

One application of such a model is to provide a naming convention for every metadata considered within the model, in order to be able to identify one concept in various models or serialisations. The idea is that by navigating in the model following the logical links provided, it is possible to construct identifiers called Utypes that could be understood by any VO tool aware of the model. To avoid multiplicity, the Utypes are built from the XML schema representation of the model which already enforces a hierarchical structure. For instance, the size of the sampling element along the spatial axis in a 2D image corresponds to: Characterisation.spatialAxis.samplingPrecision.samplingPrecisionRefVal.sampleExtent The full list of Utypes derived from this model (versions 1.1x of the model) is stored at http://www.ivoa.net/Documents/latest/UtypeListCharacterisationDM.html

\subsubsection{VOTABLE serialisation}

A VOTABLE serialisation of the characterisation of the IFU MPFS data set is shown in Appendix C. Each CharacterisationAxis is shown as a table, where each property itself is shown as a Group of FIELDS. UML class attributes are serialised as FIELDS (except if they have a detailed STC structure; in that case they are translated as a group of FIELDS). In this example, Utypes are set for each Table, Group, and Field according to the following rule:

A Utype is elaborated for each VOTable item in the serialisation as a string based on instance variable paths in our object-oriented datamodel.

Other ways of deriving utypes from a valid Xpath to the equivalent XML element in the XML Characterisation schema have been studied. The main difference is that this option may use constrained element (or attribute) values in the Utype path. The IVOA needs to define a single and robust rule to define this concept.

\section{A Appendix A: XML serialisation example}

An XML instance document representing the characterisation of an IFU data set, taken with the Russian MPFS instrument. It relies on the XML schema mentioned above. See the corresponding XML document at :

http://www. ivoa.net/internal/IVOA/CharacterisationDataModel/MPFS-v1.11.xml.

\section{B Appendix B: VOTable serialisation example}

An alternative serialisation, using the VOTable format and applying the Utype mechanism to map the various items to the Characterisation Data Model classes and attributes. Utypes are derived from the Characterisation XML schema as mentioned above. See the full XML document at :

http://www.ivoa.net/internal/IVOA/CharacterisationDataModel/MPFSVDt-v1.11.xml 
C Appendix C: Characterisation of various dataset properties 


\begin{tabular}{|c|c|c|c|c|}
\hline $\begin{array}{l}\text { AXES } \\
\text { Properties }\end{array}$ & SPATIAL & TEMPORAL & SPECTRAL & $\begin{array}{l}\text { OBSERVABLE } \\
\text { E.G. FLUX }\end{array}$ \\
\hline \multicolumn{5}{|l|}{ Coverage } \\
\hline Location & $\begin{array}{l}\text { Central } \\
\text { position }\end{array}$ & Mid-time & $\begin{array}{l}\text { Central } \\
\text { wavelength }\end{array}$ & Average flux \\
\hline Bounds & $\begin{array}{l}\text { RA, Dec [min, } \max ] \\
\text { or Bounding box } \\
\text { [center, size] }\end{array}$ & $\begin{array}{l}\text { Start/stop } \\
\text { time }\end{array}$ & $\begin{array}{l}\text { Wavelength } \\
{[\text { min, max }]}\end{array}$ & $\begin{array}{l}\text { Saturation, } \\
\text { Limiting flux }\end{array}$ \\
\hline Support & $\begin{array}{l}\text { FOV as } \\
\text { array of polygons }\end{array}$ & $\begin{array}{l}\text { Time intervals } \\
\text { (array) }\end{array}$ & $\begin{array}{l}\text { Wavelength } \\
\text { intervals } \\
\text { (array) }\end{array}$ & \\
\hline Sensitivity & $\begin{array}{l}\text { Quantum efficiency } \\
(\mathrm{x}, \mathrm{y})\end{array}$ & & $\begin{array}{l}\text { Transmission } \\
\text { curve }(\lambda)\end{array}$ & $\begin{array}{l}\text { Function } \\
\text { property } \\
\text { e.g. linearity }\end{array}$ \\
\hline $\begin{array}{l}\text { Filling } \\
\text { factor }\end{array}$ & $\begin{array}{l}\text { Effective/ } \\
\text { Total area }\end{array}$ & $\begin{array}{l}\text { Live time } \\
\text { fraction }\end{array}$ & & \\
\hline Resolution & $\begin{array}{l}\text { PSF (x,y) } \\
\text { or its FWHM }\end{array}$ & $\begin{array}{l}\text { Duration } \\
\text { per image }\end{array}$ & $\begin{array}{l}\text { Band } \\
\text { FWHM }\end{array}$ & $\begin{array}{l}\text { Flux SNR } \\
\text { (stat error) }\end{array}$ \\
\hline $\begin{array}{l}\text { Sampling } \\
\text { Precision }\end{array}$ & $\begin{array}{l}\text { Pixel scale } \\
(\mathrm{x}, \mathrm{y})\end{array}$ & $\begin{array}{l}\text { Duration } \\
\text { per image }\end{array}$ & $\begin{array}{l}\text { Band } \\
\text { FWHM }\end{array}$ & $\begin{array}{l}(1 \mathrm{ADU} \\
\text { equivalent }= \\
\text { Quantization })\end{array}$ \\
\hline
\end{tabular}

Table 2: Property versus Axis description of metadata describing a $2 \boldsymbol{D}$ optical image. This represents a single integration or indivisible stack of exposures, taken in a single broad-band filter, so the spectral resolution is the same as the filter FWHM. 


\begin{tabular}{|c|c|c|c|c|}
\hline $\begin{array}{l}\text { AXES } \\
\text { Properties }\end{array}$ & SPATIAL & TEMPORAL & SPECTRAL & $\begin{array}{l}\text { OBSERVABLE } \\
\text { E.G. FLuX }\end{array}$ \\
\hline \multicolumn{5}{|l|}{ Coverage } \\
\hline Location & $\begin{array}{l}\text { Central } \\
\text { position }\end{array}$ & Mid-Time & $\begin{array}{l}\text { Central } \\
\text { wavelength }\end{array}$ & $\begin{array}{l}\text { Average } \\
\text { flux }\end{array}$ \\
\hline Bounds & $\begin{array}{l}\text { Slit RA, Dec } \\
\text { [min, max] or } \\
\text { Bounding box }\end{array}$ & Start/stop time & $\begin{array}{l}\text { Wavelength } \\
{[\min , \max ]}\end{array}$ & $\begin{array}{l}\text { Saturation, } \\
\text { Limiting } \\
\text { flux }\end{array}$ \\
\hline Support & $\begin{array}{l}\text { Slit as accurate } \\
\text { array of } \\
\text { polygons }\end{array}$ & $\begin{array}{l}\text { Time } \\
\text { (intervals) } \\
\text { (array) }\end{array}$ & $\begin{array}{l}\text { Wavelength } \\
\text { intervals } \\
\text { (array) }\end{array}$ & $\begin{array}{l}\text { Lowest and } \\
\text { highest } \\
\text { value }\end{array}$ \\
\hline Sensitivity & $\begin{array}{l}\text { Response }(\mathrm{x}, \mathrm{y}) \\
\text { along slit }\end{array}$ & & $\begin{array}{l}\text { Quantum } \\
\text { efficiency } \\
(\lambda)\end{array}$ & $\begin{array}{l}\text { Function } \\
\text { property } \\
\text { e.g. Linearity }\end{array}$ \\
\hline $\begin{array}{l}\text { Filling } \\
\text { factor }\end{array}$ & $\begin{array}{l}\text { Effective/ } \\
\text { Total area }\end{array}$ & $\begin{array}{l}\text { Live time } \\
\text { fraction }\end{array}$ & & \\
\hline Resolution & $\begin{array}{l}\text { Slit } \\
\text { area }\end{array}$ & $\begin{array}{l}\text { Min. extractable } \\
\text { interval }\end{array}$ & $\begin{array}{l}\text { LSF or its } \\
\text { FWHM }\end{array}$ & $\begin{array}{l}\text { FluxSNR } \\
\text { (stat error) }\end{array}$ \\
\hline $\begin{array}{l}\text { Sampling } \\
\text { Precision }\end{array}$ & $\begin{array}{l}\text { Slit } \\
\text { area }\end{array}$ & $\begin{array}{l}\text { Min. extractable } \\
\text { interval }\end{array}$ & $\begin{array}{l}\text { Pixel scale } \\
\text { in } \lambda\end{array}$ & $\begin{array}{l}\text { (1 ADU } \\
\text { equivalent } \\
\text { Quantization) }\end{array}$ \\
\hline
\end{tabular}

Table 3: Property versus Axis description of metadata describing a 1DSpectrum. 


\begin{tabular}{|c|c|c|c|c|}
\hline $\begin{array}{l}\text { AXES } \\
\text { Properties }\end{array}$ & SPATIAL & TEMPORAL & SPECTRAL & $\begin{array}{l}\text { OBSERVABLE } \\
\text { E.G. FLUX }\end{array}$ \\
\hline \multicolumn{5}{|l|}{ Coverage } \\
\hline Location & $\begin{array}{l}\text { Central } \\
\text { position }\end{array}$ & Mid-Time & $\begin{array}{l}\text { Central } \\
\text { wavelength } \\
\text { (all spectra) }\end{array}$ & $\begin{array}{l}\text { Average } \\
\text { flux }\end{array}$ \\
\hline Bounds & $\begin{array}{l}\text { Field } \\
\text { RA, Dec } \\
{[\min , \max ]}\end{array}$ & $\begin{array}{l}\text { Start/stop } \\
\text { time }\end{array}$ & $\begin{array}{l}\text { Wavelength } \\
\text { [min,max] } \\
\text { (all spectra) }\end{array}$ & $\begin{array}{l}\text { Saturation, } \\
\text { Limiting } \\
\text { flux }\end{array}$ \\
\hline Support & $\begin{array}{l}\text { Union of fiber } \\
\text { footprints } \\
\text { on the sky }\end{array}$ & $\begin{array}{l}\text { Time } \\
\text { intervals } \\
\text { (array) }\end{array}$ & $\begin{array}{l}\text { Disjoint } \\
\text { wavelength } \\
\text { intervals }\end{array}$ & $\begin{array}{l}\text { Lowest and } \\
\text { highest } \\
\text { value }\end{array}$ \\
\hline Sensitivity & $\begin{array}{l}\text { Response }(\mathrm{x}, \mathrm{y}) \\
\text { along } \\
\text { the slit }\end{array}$ & & $\begin{array}{l}\text { Quantum } \\
\text { efficiency } \\
(\lambda)\end{array}$ & $\begin{array}{l}\text { Function } \\
\text { property } \\
\text { e.g. Linearity }\end{array}$ \\
\hline $\begin{array}{l}\text { Filling } \\
\text { factor }\end{array}$ & $\begin{array}{l}\text { Effective/ } \\
\text { Total area }\end{array}$ & $\begin{array}{l}\text { Live time } \\
\text { fraction }\end{array}$ & & \\
\hline Resolution & $\begin{array}{l}\text { PSF (x,y) } \\
\text { or its } \\
\text { FWHM }\end{array}$ & $\begin{array}{l}\text { Min. } \\
\text { extractable } \\
\text { interval }\end{array}$ & $\begin{array}{l}\text { LSF } \\
\text { or its } \\
\text { FWHM }\end{array}$ & $\begin{array}{l}\text { Flux } \text { SNR } \\
\text { (stat error) }\end{array}$ \\
\hline $\begin{array}{l}\text { Sampling } \\
\text { Precision }\end{array}$ & $\begin{array}{l}\text { Pixel scale } \\
(\mathrm{x}, \mathrm{y})\end{array}$ & $\begin{array}{l}\text { Min. } \\
\text { extractable } \\
\text { interval }\end{array}$ & $\begin{array}{l}\text { Pixel } \\
\text { scale } \\
\text { in } \lambda\end{array}$ & $\begin{array}{l}\text { (1 ADU } \\
\text { equivalent } \\
\text { Quantization) }\end{array}$ \\
\hline
\end{tabular}

Table 4: Property versus Axis description of metadata describing $3 \boldsymbol{D} \boldsymbol{I F U}$ data. These are taken using a mask of multiple slits or fibres each focusing a separate spectrum onto a single detector array. The Support comprises multiple discrete intervals in all dimensions, into which data products could be decomposed. The spatial resolution is determined by the telescope aperture (and the seeing) which spreads the incident radiation over several CCD pixels; the resolution and pixel scales impose different constraints on downstream data analysis. 


\begin{tabular}{|c|c|c|c|c|}
\hline $\begin{array}{l}\text { AXES } \\
\text { Properties }\end{array}$ & SPATIAL & TEMPORAL & SPECTRAL & $\begin{array}{l}\text { OBSERVABLE } \\
\text { E.G. Flux }\end{array}$ \\
\hline \multicolumn{5}{|l|}{ Coverage } \\
\hline Location & $\begin{array}{l}\text { Central } \\
\text { position }\end{array}$ & Mid- Time & $\begin{array}{l}\text { Central } \\
\text { Frequency }\end{array}$ & Average flux \\
\hline Bounds & $\begin{array}{l}\text { RA,Dec }[\min , \max ] \\
\text { or Bounding box } \\
\text { [center, size] }\end{array}$ & $\begin{array}{l}\text { Start/stop } \\
\text { time }\end{array}$ & $\begin{array}{l}\text { Frequency } \\
{[\min , \max ]}\end{array}$ & $\begin{array}{l}\text { Saturation, } \\
\text { rms noise }\end{array}$ \\
\hline Support & $\begin{array}{l}\text { Primary beam } \\
\text { FWHM } \\
\text { (or mosaic polygons) }\end{array}$ & $\begin{array}{l}\text { Time intervals } \\
\text { (array) }\end{array}$ & $\begin{array}{l}\text { Frequencies } \\
\text { (array) }\end{array}$ & $\begin{array}{l}\text { Peak, } \\
3 \sigma \mathrm{rms}\end{array}$ \\
\hline Sensitivity & $\begin{array}{l}\text { Smearing limits/ } \\
\text { functions (of integ. } \\
\text { time/ chan. width) }\end{array}$ & $\begin{array}{l}\text { Gain- } \\
\text { elevation }\end{array}$ & $\begin{array}{l}\text { Bandpass } \\
\text { function }(\mathrm{s}) \\
\text { or FWHM(s) }\end{array}$ & $\begin{array}{l}\text { Dynamic } \\
\text { range }\end{array}$ \\
\hline $\begin{array}{l}\text { Filling } \\
\text { factor }\end{array}$ & $\begin{array}{l}\text { Fraction } \\
\text { of mosaic } \\
\text { filled }\end{array}$ & $\begin{array}{l}\text { Live time } \\
\text { fraction }\end{array}$ & $\begin{array}{l}\text { Fraction } \\
\text { above FWHM } \\
\text { sensitivity }\end{array}$ & \\
\hline Resolution & $\begin{array}{l}\text { Spatial scales } \\
\text { (max and min of } \\
\text { BMaj, BMin, BPA) }\end{array}$ & $\begin{array}{l}\text { Min. imageable } \\
\text { duration }\end{array}$ & $\begin{array}{l}\text { FWHM of } \\
\text { Hanning } \\
\text { smoothing }\end{array}$ & RMS noise \\
\hline $\begin{array}{l}\text { Sampling } \\
\text { Precision }\end{array}$ & $\begin{array}{l}\text { Pixel scales } \\
{[\text { min, max] }}\end{array}$ & $\begin{array}{l}\text { Integration } \\
\text { time }\end{array}$ & $\begin{array}{l}\text { Channel } \\
\text { width }\end{array}$ & \\
\hline
\end{tabular}

Table 5: Property versus Axis description of metadata describing a radio image service, potentially mosaiced. The Max. and Min. spatial resolutions arise from the shortest and longest baselines present; any intermediate value may be selected when an image is extracted from visibility data. The spectral resolution may be coarsened by smoothing to minimise artefacts. 


\begin{tabular}{|l|l|l|l|l|}
\hline $\begin{array}{l}\text { AXES } \\
\text { Properties }\end{array}$ & SPATIAL & TEMPORAL & SPECTRAL & $\begin{array}{l}\text { OBSERVABLE } \\
\text { E.G. Flux }\end{array}$ \\
\hline Coverage & $\begin{array}{l}\text { Central } \\
\text { position } \\
(0,0)\end{array}$ & Mid- Time & $\begin{array}{l}\text { Central } \\
\text { Frequency }\end{array}$ & Average flux \\
\hline Location & $\begin{array}{l}\text { Bounding box } \\
\text { center, size }]\end{array}$ & $\begin{array}{l}\text { Relative } \\
\text { start/stop time }\end{array}$ & $\begin{array}{l}\text { Frequency } \\
{[\text { min,max }]}\end{array}$ & $\begin{array}{l}\text { Saturation, } \\
\text { rms noise }\end{array}$ \\
\hline Support & $\begin{array}{l}\text { FOV as array } \\
\text { of polygons }\end{array}$ & Time interval & Frequencies & \\
\hline Sensitivity & $\begin{array}{l}\text { Quantum efficiency } \\
\text { (x, y) }\end{array}$ & $\begin{array}{l}\text { Effective/ } \\
\text { Total area }\end{array}$ & $\begin{array}{l}\text { Transmission } \\
\text { curve }\end{array}$ & $\begin{array}{l}\text { Detector } \\
\text { linearity }\end{array}$ \\
\hline $\begin{array}{l}\text { Filling } \\
\text { factor }\end{array}$ & $\begin{array}{l}\text { PSF } \\
\text { FWHM }\end{array}$ & Duration & $\begin{array}{l}\text { Band } \\
\text { FWHM }\end{array}$ & $\begin{array}{l}\text { Noise } \\
\text { error }\end{array}$ \\
\hline Resolution & $\begin{array}{l}\text { Pixel scales } \\
\text { x, y }]\end{array}$ & Duration & $\begin{array}{l}\text { Band } \\
\text { FWHM }\end{array}$ & Quantization \\
\hline $\begin{array}{l}\text { Sampling } \\
\text { Precision }\end{array}$
\end{tabular}

Table 6: Property versus Axis description of metadata describing a simulated $\boldsymbol{C C D}$ observation in a single band. The spatial coordinates may be expressed in $(x, y)$ independent of celestial position. 


\section{Appendix D: Requirements for Data Model compliance}

\section{D.1 Limitations in this version}

The first three levels of Characterisation are now fully described and take explicit values. The fourth level of the structure can contain functions (e.g. the variation of noise with position) or URLs (e.g. the location of a weight map). Data providers may have varying expectations about how these advanced metadata should be delivered, so we will expand the description of this level in a future version of the model, after polling the community for the use of weight maps, variability maps, etc... We anticipate that the first three levels will answer more than $70 \%$ of present needs.

It is not yet possible to implement rules linking coverage on different axes. For example, if a survey consists of spatially distinct fields, observed in several wavebands, but there are fields which do not contain all wavebands, then each field and/or each waveband must be described separately. Similarly, separate descriptions are required if resolution or noise (for instance) behave differently in various areas of Support.

\section{D.2 Implementing Characterisation}

\section{D.2.1 Data Providers}

Several tools are being developed to assist data providers supply metadata. These include extraction of information from FITS headers and a form interface called CAMEA which allows the user to enter values for Characterisation elements and translates this to XML. We will also provide XML templates for manual editing. We will investigate what would be more convenient for large data collections depending on how they store their existing metadata.

Metadata required by Characterisation might be extracted from a number of sources such as:

- An archive database;

- An observing log or other description which might be stored in a database or as ascii, xml or other documents;

- FITS headers, which provide more or less direct routes:

- Unambiguous identification between e.g. a database column or FITS keyword and a Characterisation element;

- Correspondence with formulaic modification, e.g. adding explicit units or calculating the field of view of an interferometer;

- A separate information source e.g. resolution of the telescope using different frequencies/configurations;

- Offine/human memory/judgement

The following sections outline our proposals for which of the status strings MANDATORY, RECOMMENDED or OPTIONAL should be applied to each element of the XML schema. The status strings are used as in the SIAP proposed recommendation (http: //www.ivoa.net/Documents/WD/SIA/sia-20040524.html), interpreted as follows:

- MANDATORY means that the metadatum is fully required to make the data usable in basic VO services 
- RECOMMENDED means that this item should be given if at all possible to improve the interpretation of the data or their use in a wider range of VO services

- OPTIONAL means that such metadata elements would help to give more precise interpretation but are not vital.

An implementation may have one among the following level of compliance and be :

- "partially compliant", if it implements some (but not all) MANDATORY/MUST elements

- "compliant", if it implements all MANDATORY/MUST elements

- "fully compliant", if it implements all MANDATORY/MUST elements and all RECOMMENDED/SHOULD ones

The prime goal is to get this model applied by data providers in useful ways. We should make it as easy as possible to describe any kind of observed or simulated data by minimising the number of compulsory fields. At the same time we must encourage data providers to give enough information to expand the ways in which data can be selected or manipulated by VO tools currently or imminently available.

\section{D.3 Requirements for compliance}

\section{D.3.1 General considerations}

On each axis, the first three levels (Location, Bounds, Support), must be given explicit numerical values (or arrays of values) in order to be accessible to any tool. Other elements may be given numerical values, or functions, or indirect references (URIs) but these are in general not used at present.

Users are strongly encouraged to evaluate coarser levels of description explicitly even if they also provide finer levels. We need to decide what users do if they are not giving a value for an element e.g. leave blank, consistent with other models.

Location, Bounds and other higher Characterisation levels are intentionally approximations to provide a simple inclusive description of the data.

The Location value may be determined with some error, which might be mentioned inside the STC structure used for Coordinates. This is to be distinguished from Bounds which should be the outer limits to anywhere data might be found.

Accuracy properties describe uncertainties in the mapping process of data values along axes, see Section D.7.

The values for some elements must be given as arrays, defined as in STC, and the required number of arguments must be present if any are. Bounds, for instance, describes a unique region on an axis as e.g. $(\alpha 1, \delta 1 ; \alpha 2, \delta 2)$, whilst the ResolutionSupport is given relatively e.g. telescope beam major and minor axes in arcsec and position angle.

\section{D.3.2 Defaults}

Defaults might sometimes be possible for values which have not been provided. We do not think that such defaults should be coded into the description, rather that software which looks for the value of a missing element might be able to make an intelligent assumption. It is up to the writers of a software tool specification to decide whether it is more dangerous to use defaults and risk a lower level of accuracy, or to ignore data which is not adequately specified and thus loose potentially important information. 
For example, if Location is not given then, for some axes, software may take the default Location as the mid-point of the Bounds ${ }^{7}$.

If Bounds are not given then e.g. if a spatial axis has Coordsys ICRF some software might assume all-sky coverage ${ }^{8}$.

If Support is not given Bounds, if present, could be used.

If the unit or Coordsys element is not given for any level, the values for the CharacterisationAxis are used; be careful, as this may be unsuitable (e.g. if the CharacterisationAxis units are sexagesimal, then a single number for an error could be in degrees or $\operatorname{arcsec}$ or ...).

\section{D.4 Axes}

It is MANDATORY to provide at least one axis (coded as a CharacterisationAxis element). All three of the Space, Time and Spectral Coverage axes are RECOMMENDED ${ }^{9}$.

The unit and coordinate system are MANDATORY for each Axis present. These may be relative to an internal reference only, e.g. pixel spatial coordinates. In such a case both the Location and Bounds are MANDATORY for that axis. Note that STC allows 'RELOCATABLE', for example as a valid Location for simulated data, unless this is incompatible with the specified coordinate system.

Space-, Spectral- and Time-related axes, and most other potential axes, are already defined in STC; where this is the case, it is MANDATORY to use the STC coordinate system and unit definitions. Various cases of how to re-use STC elements are shown in the example XML documents provided. The Observable Axis is RECOMMENDED ${ }^{10}$.

Axes which are not yet defined in STC (such as Polarization at the present time) are OPTIONAL but a reference to the definition of the proper Coordinate System should be given.

\section{D.4.1 Axis Flags}

For each CharacterisationAxis element (spatial, spectral, observable, etc.):

A flag to indicate if it is an independent or a dependent variable ('true' or 'false') is RECOMMENDED.

A flag to indicate its calibration status is RECOMMENDED:

CALIBRATED, UNCALIBRATED, RELATIVE, NORMALIZED; default UNCALIBRATED.

Flags to indicate SamplingStatus are OPTIONAL (these are RECOMMENDED where they are customarily relevant):

- undersamplingStatus ('true' or 'false')

- regularsamplingStatus ('true' or 'false')

\footnotetext{
${ }^{7}$ this might be complicated (e.g. some spatial coordinates) or impossible

8 a more restricted coverage might be derived once there is a link to Observation and the telescope location

${ }^{9}$ some might be considered irrelevant for simulated data, or not conventionally provided e.g. for old spectra with no time stamp

${ }^{10}$ its omission may seem reasonable for e.g. the coverage intended for a future survey
} 


\section{D.5 Coverage}

For each CharacterisationAxis, it is MANDATORY to give either the Location or the Bounds elements. Both Location and Bounds are RECOMMENDED if these are available.

Support is RECOMMENDED; if it is given then it is MANDATORY also to give the Bounds ${ }^{11}$.

Sensitivity ${ }^{12}$ (e.g. the URI of a weight map, or a function) is OPTIONAL.

The Unit and/or CoordSystem is OPTIONAL for each of these coverage layers; if not given, they will default to the units and CoordSystem used for the CharacterisationAxis element (i.e. when the axis was first defined).

\section{D.6 Other Properties: Resolution and Sampling Preci- sion}

Resolution and SamplingPrecision relate to a specific Coverage along one CharacterisationAxis. They are organised according to progressive levels of description as in Coverage but themselves contain the relevant layers, e.g., for some axis:

- at level 1: resolutionRefVal instead of Location stands for a typical or average value for the resolution as in Spectral.Resolution.resolutionRefVal

- at level 2: Bounds contains the lowest and highest values present as in Spatial.Resolution.resolutionBounds

- at level 3: Support represents sets of discrete ranges of sampling intervals as in Spectral.SamplingPrecision.Support

- at level 4: resolutionVariability stores the variability of resolution with position on the axis as in Spatial.Resolution.Variability

If there are many areas of Support within the Coverage, the Accuracy, Resolution and SamplingPrecision should refer to the inside of each Support area. However, in this version of the model, it is assumed that, in principle, on any one axis, one description of each of these properties applies to all Support areas, otherwise each area must be described in a separate Characterisation tree description (see Section D.1).

The Resolution and/or SamplingPrecision are OPTIONAL; if they are present, it is MANDATORY to give the unit and Coordsys on axes where the units of the CharacterisationAxis would not make sense or are ambiguous; otherwise the CharacterisationAxis values are used. The unit and Coordsys are OPTIONAL for any level of Accuracy, Resolution or Sampling, otherwise the value defined at the start of the Accuracy, Resolution or Sampling definitions is used.

\footnotetext{
${ }^{11}$ If different areas of Support apply on different axes, a separate description should be used at the level where each subset of data can be described unambiguously, see Section D.1

${ }^{12}$ Here, Sensitivity is the dependence of a detector response or equivalent with position on the given axis. This is not the limiting sensitivity in the sense of the faintest detectable flux, which is given by the lower Bound of the Observable axis.
} 


\section{D.6.1 Resolution}

If Resolution is present, then it is MANDATORY to give the ResolutionRefVal (i.e. Location). ResolutionBounds are RECOMMENDED. The ResolutionSupport. and ResolutionVariability (as a function of position on that axis) are OPTIONAL.

\section{D.6.2 Sampling Precision}

If SamplingPrecision is present, it is MANDATORY to give a samplingPrecisionRefVal (i.e. Location) which contains both samplingPeriod and sampleExtent. It is MANDATORY to provide the samplingPeriod, whilst an explicit sampleExtent is RECOMMENDED but it is not required.

SamplingPrecisionBounds, SamplingPeriodLimits and sampleExtentLimits are also RECOMMENDED.

The SamplingPrecision.Support and related values for the samplingPeriod and/or the sampleExtent are OPTIONAL. The samplingPrecision.Variability (i.e. Sensitivity) (in the form of a samplingPrecisionMap to describe variations along an axis) is OPTIONAL.

The FillFactor is RECOMMENDED for any axis where the actual coverage in each Support region is significantly less than 1 but the filling is too complex to be described practically using Sampling ${ }^{13}$.

The FillFactor of the SamplingPrecison is OPTIONAL; if it is present and if SamplingPeriod and SampleExtent are also given, then logically:

FillFactor $=$ SampleExtent/SamplingPeriod

and the data provider should take care that the values and units given are consistent with this relationship.

\section{D.7 Accuracy}

Accuracy values for the precision of measurements are RECOMMENDED for each CharacterisationAxis, divided into statistical and systematic uncertainties (or appropriate alternative definitions of uncertainties). For each CharacterisationAxis where Accuracy is provided:

- It is MANDATORY to give the unit and Coordsys on axes where the units of the CharacterisationAxis would not make sense or are ambiguous, otherwise the CharacterisationAxis values are used.

- The unit and Coordsys are OPTIONAL for any axis ${ }^{14}$.

- It is MANDATORY to give the ErrorRefVal (typical value).

- The ErrorBounds are OPTIONAL for uncertainties which vary along the domain of the axis.

- The URI of an ErrorMap which describes the variation of errors with location is OPTIONAL.

\footnotetext{
${ }^{13}$ FillFactor applies to the usable fraction of data within each Support area, as presently defined. If we find that the majority of users want it to be the useful fraction of the whole Bounds, the name and definition will be changed in a future version.

${ }^{14}$ for example normalised units such as a flux accuracy of 0.03 given flux measurement.
} 


\section{E Appendix E: Updates of this document}

- version 0.9

- full re-organisation, rewriting and simplification of the Model description, Anita Richards April/May 2006

- new XML schema, F.Bonnarel

- version 0.93

- update XML schema and use STC blocs elements

- add examples of characterisation metadata for various data sets and various dimensions

- version 0.96 to 0.99

- finalise the XML schema and interface it with the STC XML schema

- update the document format according to the IVOA master document.

- version 1.0

- revision by the authors. Fill factor, Support discussions: minor formulation changes

- add Appendix D: Requirements for Data Model compliance prepared by Anita Richards and discussed at the Moscow interoperability meeting

- version 1.1

- modify data model for more compatibility to Spectrum data model : remove AxisFrame class not present in Spectrum DM.

- modify schema and figures accordingly

- version 1.11

- make the document compatible to IVOA referencing mecanism: update links.

- list up changes of the various draft versions in the present document

- version 1.12

Changes following the RFC and tcg comments and requirements

- Schema reference:update links to XML schema

http://www . ivoa.net/xml/Characterisation-v1.11

- update ref to examples MPFS :

http://alinda.u-strasbg.fr/Model/Characterisation/examples/MPFS-v1.11.xml

- change Fig.1 about interactions between Characterisation and other models. Observation class gets more obvious and inclusion of Char inside Observation DM too.

- update reference to the Quantity DM: simplify

- introduce Spectrum DM reference in section 2.3 'Links to other efforts'

- include a Scope section at the beginning of the document

- Appendix E: include the document revision history directly in this file instead of pointing to an external file

- use smaller fonts for figure captions

March 2008, 25: corrected the links to the UtypeList document 